\title{
Effect of sensor noise on the resolution and spatial resolution of displacement and strain maps estimated with the grid method
}

\author{
Michel GrédiaC* and Frédéric SuR ${ }^{\dagger}$
}

This is the accepted version of the following article:

M. Grédiac and F. Sur. Effect of sensor noise on the resolution and spatial resolution of displacement and strain maps estimated with the grid method. Strain, volume 50, issue 1, pages 1-27, February 2014 (50th anniversary article)

which has been published in final form at:

http://onlinelibrary.wiley.com/doi/10.1111/str.12070/abstract

\begin{abstract}
This paper deals with noise propagation from camera sensor to displacement and strain maps when the grid method is employed to estimate these quantities. It is shown that closed-form equations can be employed to predict the link between metrological characteristics such as resolution and spatial resolution in displacement and strain maps on the one hand, and various quantities characterizing grid images such as brightness, contrast and standard deviation of noise on the other hand. Various numerical simulations confirm first the relevance of this approach in the case of an idealised camera sensor impaired by a homoscedastic Gaussian white noise. Actual CCD or CMOS sensors exhibit however a heteroscedastic noise. A pre-processing step is therefore proposed to first stabilise noise variance prior to employing the predictive equations, which provide the resolution in strain and displacement maps due to sensor noise. This step is based on both a modelling of sensor noise and the use of the generalised Anscombe transform to stabilise noise variance. Applying this procedure in the case of a translation test confirms that it is possible to model correctly noise propagation from sensor to displacement and strain maps, and thus also to predict the actual link between resolution, spatial resolution and standard deviation of noise in grid images.
\end{abstract}

Keywords: digital image correlation, displacement measurement, generalised Anscombe transform, grid method, metrology, noise, noise characterisation, resolution, spatial resolution, strain measurement

\footnotetext{
${ }^{*}$ Institut Pascal, UMR CNRS 6602, Clermont Université, Université Blaise Pascal, BP 10448, 63000 Clermont-Ferrand, France.

${ }^{\dagger}$ LorIA, UMR 7503, Université de Lorraine, CNRS, INRIA projet Magrit, Campus Scientifique, BP 239, 54506 Vandoeuvre-lès-Nancy Cedex, France.
} 


\section{Introduction}

Full-field measurement techniques are increasingly used in the experimental mechanics community although their actual metrological performance have not been precisely quantified so far. One of the main reasons is that measurement fields such as displacement or strain fields are considered instead of mere scalar quantities. This leads some metrological characteristics to be dependent on the others but the link between them is not clearly established in the literature. In addition, displacement is in general the raw physical quantity provided by full-field measurement systems but strain is often the final desired quantity since constitutive equations which are identified with such systems relate stress and strain. Strain components being deduced from displacements by differentiation, this leads to choose some additional parameters for the numerical implementation of strain estimation, thus making it difficult to have a clear overview on the influence of each of the numerous quantities that finally influence strain maps obtained with a given measuring system.

In practice, two metrological characteristics are often considered: resolution and spatial resolution. Resolution can be defined by the change in quantity being measured that causes a change in the corresponding indication greater than one standard deviation of the measurement noise [10]. Note that, while taking one standard deviation instead of any multiple is quite arbitrary, the idea is that the resolution quantifies the smallest change not likely to be caused by the measurement noise. Spatial resolution is defined here by the shortest distance between two spatially independent measurements. Since Gaussian windows are used in the approach employed here (other windows could be employed, but this point is not discussed here), it seems reasonable to define the spatial resolution by a multiple of the standard deviation of the Gaussian window. This definition holds here for both the displacement and the strain since it can be shown that these two quantities can be determined separately $[4,5]$. The "smoothness" of the obtained strain maps is directly driven by the width of the Gaussian window which appears in the windowed Fourier transform (WFT) employed in this study to process the grid images, as explained below. This reduces to one the number of parameters chosen by the user which influence the strain maps for a set of grid images, thus making it easier to study its influence on these maps.

It is well-known that the resolution and the spatial resolution of any full-field measurement method evolve in a contradictory way: the higher the resolution, the lower the spatial resolution and vice versa. It is therefore not possible to provide a unique value for each of these quantities for a given measurement system. Providing a relationship between resolution and spatial resolution is instead more relevant. This issue is addressed in various studies dealing with digital image correlation $[33,39,38,21]$ for instance. Similar studies concerning error assessment in stereo-based deformation measurements are also available [40, 22]. The link between resolution, spatial resolution and actual noise in digital images is generally not given, or only global and averaged esti- 
mates are provided. Moreover, digital noise is more complicated than a mere Gaussian white noise as illustrated in this paper. Some papers also investigate noise propagation in full-field measurement techniques, using for instance a Monte Carlo approach [25, 32] but a recent review paper confirms that noise is only scarcely taken into account in the assessment of the measurement uncertainty of full-field measurement techniques based on phase analysis [18].

In this context, the present work is aimed at providing a clear relationship between noise in grid images provided by a camera, and some metrological characteristics of the grid method, namely its resolution and spatial resolution, the latter being directly proportional to the window in the WFT employed to extract the phases and their derivatives. Resolution and spatial resolution will be considered separately for both displacement and strain. The relevance of this relationship will be illustrated through some numerical simulations and experiments.

The paper is organised as follows. The basics of the measuring method employed here, namely the grid method, are first recalled in Section 2. The main advantage is to rely in this case on quasi-regular marking of the surface under investigation, thus making it possible to derive predictive closed-form expressions between noise in the grid images, resolution and spatial resolution for phase, phase derivative, displacement and strain maps (Sections 3). These relationships are then validated through some numerical simulations in Section 4. A real case is finally addressed in Section 5. Technical details are given in three separate appendices.

\section{Determining in-plane displacement and strain compo- nents from grid images}

The objective here is to briefly recall the procedure used to determine in-plane strain components from grid images. In such images, the light intensity $s(x, y)$ at each point $(x, y)$ is a quasi-periodic signal which can be modelled as follows [5]

$$
s(x, y)=\frac{A}{2}\left(2+\gamma \cdot \operatorname{frng}\left(2 \pi f x+\phi_{1}(x, y)\right)+\gamma \cdot \operatorname{frng}\left(2 \pi f y+\phi_{2}(x, y)\right)\right)
$$

where:

- $A$ is the average global field illumination;

- $\gamma$ is the contrast of the oscillatory pattern between 0 and 1 ;

- frng is a real $2 \pi$ periodic function with a peak-to-peak amplitude equal to 1 and average value 0 ;

- $f$ is the frequency of the carrier, defined as the inverse of the pattern pitch $p$ (that is, the inter-line distance); 
- $\phi_{x}(x, y)$ and $\phi_{y}(x, y)$ are the carrier phase modulations along the $x$ - and $y$-axes respectively.

Parameters $A$ and $\gamma$ are assumed to be constant here but they may slightly change in practice in actual images. The important point is however that this change remains small at the scale of the kernel of the WFT discussed below.

The phase changes between two images, denoted $\Delta \Phi_{x}$ and $\Delta \Phi_{y}$, are related to the in-plane displacements $u_{x}$ and $u_{y}$ through the following equations [37]

$$
\left\{\begin{array}{l}
u_{x}=-\frac{p}{2 \pi} \times \Delta \Phi_{x} \\
u_{y}=-\frac{p}{2 \pi} \times \Delta \Phi_{y}
\end{array}\right.
$$

The in-plane strain components are therefore deduced from the phase derivative variations using the following equations ${ }^{1}$

$$
\left\{\begin{array}{l}
\epsilon_{x x}=-\frac{p}{2 \pi} \times \Delta \frac{\partial \Phi_{x}}{\partial x} \\
\epsilon_{y y}=-\frac{p}{2 \pi} \times \Delta \frac{\partial \Phi_{y}}{\partial y} \\
2 \epsilon_{x y}=-\frac{p}{2 \pi} \times\left(\Delta \frac{\partial \Phi_{x}}{\partial y}+\Delta \frac{\partial \Phi_{y}}{\partial x}\right)
\end{array}\right.
$$

Considering a given grid picture, the phases can be estimated by calculating a which writes as follows

$$
\begin{aligned}
\Psi(x, y, \theta) & =\int_{-\infty}^{+\infty} \int_{-\infty}^{+\infty} s(\xi, \eta) g_{\sigma}(x-\xi, y-\eta) e^{-2 i \pi f(\xi \cos (\theta)+\eta \sin (\theta))} \mathrm{d} \xi \mathrm{d} \eta \\
& =R(x, y, \theta)+i J(x, y, \theta)
\end{aligned}
$$

where $R$ and $J$ are the real and imaginary parts of $\Psi$ respectively, and $g_{\sigma}$ is a $2 \mathrm{D}$ window function of width $\sigma$. It is symmetric, positive, and integrates to 1 . In practice, this window can be a triangle, as suggested in [37], or a Gaussian, as in [5] to ensure differentiability. A Gaussian function is used in the current work. Note that a Gaussian function reduces the influence of the harmonics of the periodic frng function and also minimises the effect of noise, as demonstrated in [36], because of its fast decay in the frequency domain unlike, e.g., a triangle window function. The parameter $\theta$ can be either equal to 0 or $\frac{\pi}{2}$, depending on the direction under consideration: $x$ or $y$, respectively. The actual phase variations $\Delta \Phi_{x}(x, y)$ and $\Delta \Phi_{y}(x, y)$ are estimated by calculating the following quantities denoted $\Delta \widetilde{\Phi_{x}}(x, y)$ and $\Delta \widetilde{\Phi_{y}}(x, y)$

\footnotetext{
${ }^{1}$ There is a typo in the Strain paper; it is corrected here.
} 


$$
\left\{\begin{array}{l}
\Delta \widetilde{\Phi_{x}}(x, y)=\Delta \arctan \left(\frac{J(x, y, 0)}{R(x, y, 0)}\right) \\
\Delta \widetilde{\Phi_{y}}(x, y)=\Delta \arctan \left(\frac{J(x, y, \pi / 2)}{R(x, y, \pi / 2)}\right)
\end{array}\right.
$$

It is shown in $[36,34]$ that these quantities are actually first order approximations of the convolution of the actual phase changes by the envelope of the kernel used during the WFT, but this point is not considered in the present study.

\section{Noise propagation}

\subsection{Phase and phase derivative maps}

An important question is to know how noise propagates from the sensor of the camera to displacement and strain maps. Since these quantities are proportional to the phase and the phase derivative changes between current and reference images (see Equation 3), we study first phase and phase derivatives. Results for strain and displacement are then easily deduced.

The most direct route for characterizing noise in the phase and phase derivative maps is to study the temporal distribution of the phase and phase derivative maps at all their pixels. This procedure requires however to process repeated shots of the same scene. Another possibility is to assess the spatial distribution of these quantities when the surface under interest is subjected to a rigid-body displacement such as a translation: since the phase change is theoretically constant in this case (and its derivatives rigorously null), one can study how these quantities change from one point to the other and deduce their actual spatial change. This is a classic method for DIC to characterise the noise floor in displacement maps [19] as well as for the grid method [5], even though what is often referred to as "noise" in the experimental mechanics community is actually the superimposition of actual image noise inherent to any digital acquisition system and other causes. For instance, concerning DIC, it is generally not possible to obtain a speckle which has the same characteristics over the whole surface of the tested specimen, thus unavoidably leading to spatial changes in the metrological performance. Concerning the grid method, grids generally feature some small defects such as spatial changes in their pitch or local lack of ink which locally disturb the phase and phase derivative maps, as explained in [5,6]. These maps disturbances are superimposed to phase and phase derivative changes due to noise in the grid images caused by the camera sensor itself, and eventually contribute to the noise floor in displacement and strain maps. This paper only addresses the latter phenomenon, namely sensor noise.

Ref. [36] gives a closed-form expression for the variance of the noise in the phase or the phase derivative images (denoted $v_{\phi}$ and $v_{\phi^{\prime}}$, respectively) from the variance of the noise in the grid image, assuming that: 
- The grid images are corrupted by a Gaussian white noise of variance $v_{\text {image }}$. Since its variance is constant, the white noise is called homoscedastic. Even though the assumption of homoscedastic noise is classic in a large part of the image processing literature, it will be shown in Section 4 below that it is very rough, if not false in practice when dealing with raw data from digital sensors;

- The envelope of the kernel used to extract the phase and phase derivative maps is a Gaussian function whose standard deviation is denoted $\sigma$.

In Ref. [36], this result was establish to feed the Wiener filter used to deconvolve strain images, as shown in [16]. The contribution of the present article is to investigate in more details this result in order to see how to assess the metrological performance of the grid method a priori, in other words how this performance is linked to some parameters such as lighting and noise in the images due to the camera only. Such a result is expected to be very useful to better understand how noise propagates from grid images to the final displacement and strain maps, and ultimately to tackle each cause with a suitable tool to enhance strain images. The latter objective is however quite ambitious and out of the scope of the present paper solely devoted to noise propagation.

Displacement and strain maps are respectively proportional to phase and phase derivative changes, as recalled in Equation 2 and 3. In [36], the expression of the variance $v_{\phi}$ in the phase distribution as a function of the variance $v_{\text {image }}$ in the grid image has been established under the assumptions recalled above. In this reference, similar results are obtained concerning the relationship between the variance $v_{\phi^{\prime}}$ in the phase derivative distribution and $v_{\text {image }}$. For the sake of completeness, the calculation is briefly recalled in Appendix A. These expressions are:

$$
\left\{\begin{array}{l}
v_{\phi}=\frac{v_{\text {image }} \Delta_{x} \Delta_{y}}{8 \pi \sigma^{2} K^{2}} \text { for phase } \\
v_{\phi^{\prime}}=\frac{v_{\text {image }} \Delta_{x} \Delta_{y}}{16 \pi \sigma^{4} K^{2}} \text { for phase derivative }
\end{array}\right.
$$

where $\Delta_{x} \times \Delta_{y}$ represents the pixel size and $K$ the modulus $|\Psi|$ of the WFT of the grid image. Depending on the direction which is considered for the phase $(x$ or $y)$, $\theta=0$ or $\theta=\frac{\pi}{2}$ should be considered in Equation 4 for the calculation of $K$, but it is observed in practice and justified by the theory in [36] that the moduli obtained in both cases are very similar. From a formal point of view, no distinction is made below in the notation for quantities obtained for directions $x$ or $y$, but it is clear that the correct modulus shall be taken in the calculations in practice, depending on the direction which is considered. For instance, when the $x$-direction is considered (the $y$-direction, respectively), $K$ is the modulus $|\Psi|$ of the WFT defined in Equation 4 in which $\theta=0\left(\theta=\frac{\pi}{2}\right.$, respectively). An important remark is that $K$ is practically 
spatially constant across the maps, or at least within the Gaussian window $g_{\sigma}$, which justifies the homoscedasticity assumption. This quantity is demonstrated in [36] to be equal to

$$
K=\frac{\left|d_{1}\right| \gamma A}{2}
$$

where $\left|d_{1}\right|$ is the modulus of the coefficient of the first non-constant term in the Fourier expansion of the frng function defined in Equation 1. Note also that the noise in the phase and phase derivative maps is actually spatially correlated and the covariance is calculated in [36], but this quantity is not considered here for the sake of simplicity.

The resolution and the spatial resolution of any full-field measurement method evolve in a contradictory way: the higher the resolution, the lower the spatial resolution and vice versa. Equations 6 above enable us to study in more detail the link that exists between resolution and spatial resolution. Indeed, the resolution in displacement and the resolution in strain are directly proportional to the standard deviation of the distribution of each quantity according to their definitions recalled in the introduction above. These standard deviations are denoted $\sigma_{\phi}$ and $\sigma_{\phi^{\prime}}$, with $\sigma_{\phi}=\sqrt{v_{\phi}}$ and $\sigma_{\phi^{\prime}}=$ $\sqrt{v_{\phi^{\prime}}}$. For its part, the spatial resolution of each quantity is proportional to the width of the kernel used to extract phase and phase derivatives. These spatial resolutions are denoted $d_{\phi}$ and $d_{\phi^{\prime}}$, respectively. According to the classic $3-\sigma$ rule, this width can be defined by six times the standard deviation $\sigma$ of the Gaussian envelope of the kernel. This definition is conservative, but quite arbitrary. For instance, using four times $\sigma$ instead of six times would automatically improve the metrological performance. The standard deviation of the noise in the grid images is denoted $\sigma_{\text {image }}$, with $\sigma_{\text {image }}=\sqrt{v}$ using the notations of Appendix A. Substituting all theses quantities in Equations 6 above leads to:

$$
\left\{\begin{array}{l}
\sigma_{\phi}^{2}=\frac{9 \sigma_{\text {image }}^{2} \Delta_{x} \Delta_{y}}{2 \pi d_{\phi}^{2} K^{2}} \text { for phase } \\
\sigma_{\phi^{\prime}}^{2}=\frac{81 \sigma_{\text {image }}^{2} \Delta_{x} \Delta_{y}}{\pi d_{\phi^{\prime}}^{4} K^{2}} \text { for phase derivative }
\end{array}\right.
$$

Taking the square root of the above equations and rearranging the obtained results gives 


$$
\left\{\begin{array}{l}
\sigma_{\phi} \times d_{\phi}=\left(\frac{3}{\sqrt{2 \pi}}\right) \times\left(\frac{\sqrt{\Delta_{x} \Delta_{y}}}{K}\right) \times \sigma_{\text {image }} \quad \text { for phase and } \\
\sigma_{\phi^{\prime}} \times d_{\phi^{\prime}}^{2}=\left(\frac{9}{\sqrt{\pi}}\right) \times\left(\frac{\sqrt{\Delta_{x} \Delta_{y}}}{K}\right) \times \sigma_{\text {image }} \quad \text { for phase derivative }
\end{array}\right.
$$

In these equations, $K$ and $\sigma_{\text {image }}$ are given in grey levels. $\Delta_{x}$ and $\Delta_{y}$ are the dimensions of a pixel. Dimensions being given in pixels in the problem, $\Delta_{x}$ and $\Delta_{y}$ are equal to one and therefore vanish in the equations. Hence $\sigma_{\phi^{\prime}}$ is in pixels ${ }^{-1}$ and $\sigma_{\phi}$ has not unit. Several remarks can be drawn from these equations which predict how the resolution and the spatial resolution are connected for both the phase and the phase derivative:

- Equation 9-a shows that the metrological performance of the phase extraction, characterised here by $\sigma_{\phi} \times d_{\phi}$, is driven by a relationship similar to that deduced from the Heisenberg uncertainty principle [24], in the sense that the resolution and spatial resolution cannot be simultaneously arbitrarily low. On the other hand, differentiation involved in the calculation of the phase derivative causes the spatial resolution to be squared in the left-hand side of Equation 9-b, meaning for instance that improving the spatial resolution by a factor 2 impairs the resolution by a factor 4 . A similar property has already been discussed for the global performance of DIC in $[33,21]$;

- This performance is connected to two characteristics of the grid image: the standard deviation of the noise $\sigma_{\text {image }}$ on the one hand, and the modulus of the Fourier transform $K$ on the other hand. The following points are the consequence of the present remark :

$-\sigma_{\phi} \times d_{\phi}$ and $\sigma_{\phi^{\prime}} \times d_{\phi^{\prime}}^{2}$ are proportional to $\sigma_{\text {image }}$, thus to the standard deviation of the noise that corrupts the grid images. This is an intuitive result for phase, probably not so much for phase derivative because of the square which affects $d_{\phi^{\prime}}$;

- Both products are also inversely proportional to $K$. According to the definition of this quantity recalled in Equation 7, this result means that $\sigma_{\phi} \times d_{\phi}$ and $\sigma_{\phi^{\prime}} \times d_{\phi^{\prime}}^{2}$ are all the smaller (and thus the noise effect reduced in the phase and phase derivative maps) that the brightness (driven by $A$ ) and the contrast (driven by $\gamma$ ) of the grid image are the higher, and that $\left|d_{1}\right|$ is the higher too. This is an intuitive result for the brightness and for the contrast but the phenomenon is now quantified. Concerning the influence 
of $\left|d_{1}\right|$, it means that the nature of the profile of the lines forming the grid has an impact on the noise level. The highest value for $\left|d_{1}\right|$ is obtained for a sine function: $\left|d_{1}\right|=0.50$ in this case, see Appendix B. However printing technology does not really make it possible to print such a line profile when grids feature a small pitch (say some lines per millimeter): only totally black lines can be printed in this case. For such rectangular function, the highest value for $\left|d_{1}\right|$ is obtained when the black ink covers half of the pattern pitch $\left|d_{1}\right|=\frac{1}{\pi} \simeq 0.32$ in this case, see Appendix B. This is an important remark because the phase retrieved by the WFT does not depend on the profile of the line whereas the noise level in the obtained result depends on this profile.

- $d_{\phi}$ and $d_{\phi^{\prime}}$ are distinguished here because the phase derivative can theoretically be obtained directly, without the need to estimate first the phase [5], but both quantities are often equal in practice, especially when phase derivative is deduced from the phase by numerical differentiation;

- In general, $A$ exhibits slight variations over the image while the spatial resolutions $d_{\phi}$ and $d_{\phi^{\prime}}$ are constant. The reason is that both $d_{\phi}$ and $d_{\phi^{\prime}}$ are proportional to the standard deviation $\sigma$ which drives the width of the kernel used in the WFT. Consequently the resolutions $\sigma_{\phi}$ and $\sigma_{\phi^{\prime}}$ for both the phase and the phase derivative are not constant throughout the phase and phase derivative maps. This illustrates the fact that the metrological performance of a full-field measurement system does not boil down to some constant parameters: this is actually more subtle, so using suitable equations linking physical and metrological characteristics such as Equations 9 above seems to be more relevant and closer to reality.

\subsection{Displacement and strain maps}

Equation 3 shows that displacement (respectively strain) is proportional to a difference between two phases (respectively phase derivative) maps: the current and the reference maps. Assuming that the noise processes on these two maps are statistically independent, the noise variance on the difference map is twice larger. From Equations 2 and 9, one can directly derive the following equations for these quantities:

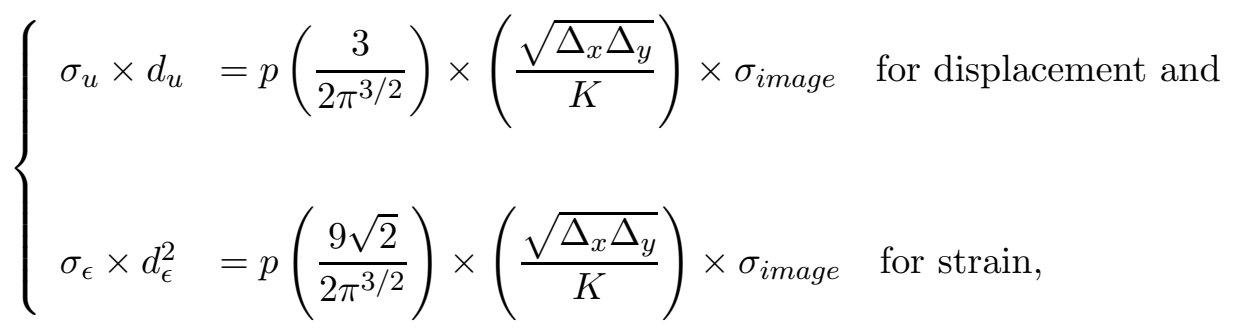


where $\sigma_{u}$ and $\sigma_{\epsilon}$ are the resolution for the displacement and strain, respectively. $d_{u}$ and $d_{\epsilon}$ are the spatial resolutions for the displacement and strain, respectively.

It is important to mention that phase maps (respectively phases derivative maps) which are subtracted to estimate displacement maps (respectively strain maps) are obtained $i$ - in the deformed geometry for the current phase (respectively phase derivative) and $i i$ - in the reference geometry for the reference phase (respectively phase derivative). Subtracting these two quantities is possible when the displacement in between remains tiny, but grids defects are generally revealed since subtraction is not performed at the same physical point. This is the reason why it is proposed to project the current map in the coordinate system of the reference map prior to subtraction in order to remove the greatest part of the flaws in the strain maps which are due to these grid defects, as suggested in [5]. Two assumptions must therefore be drawn up to employ Equations 10 to study resolution and spatial resolution in displacement and strain:

- The above-mentioned procedure has no impact on the calculation of the standard deviation, which is sound as explained in Appendix C;

- Any physical point slightly moves from the reference to the current positions, so it is not lit exactly with the same intensity from one case to each other. It is therefore necessary to assume that brightness slightly and regularly evolves over the images, so $K$ remains nearly unchanged between the reference and the current positions.

The objective now is to check whether these equations are satisfied with simulated noise. This is the aim of the next section while Section 5 is aimed at checking if they are still satisfied with experimental data.

\section{Numerical simulations}

\subsection{Image featuring spatially changing contrast and noise}

The objective here is to work on synthetic grid images corrupted by noise and to check if Equations 9 are satisfied. In these equations, the parameters related to the grid images are two: $K$, which depends on contrast $\gamma$ and illumination $A$, and the noise standard deviation $\sigma_{\text {image }}$. In practice, grid image processing is performed after choosing the size of the kernel driven by $\sigma$, so the spatial resolutions $d_{\phi}$ and $d_{\phi^{\prime}}$ are generally fixed. The idea is therefore to determine $\sigma_{\phi}$ and $\sigma_{\phi^{\prime}}$ for any set of parameters $\left(K, \sigma_{\text {image }}\right)$. Rather than considering various grid images characterised each by constant values for $\left(K, \sigma_{\text {image }}\right)$, the idea is to work with a set of synthetic grid images for which both quantities vary at the same time, each along a direction. These variations must remain small so that each of these variations remains negligible over the surface of the kernel of the WFT. Since the right-hand sides of Equations 9 are expected to remain constant 
when only changing resolution and spatial resolution and since $K$ and $\sigma_{\text {image }}$ change in this synthetic image, the idea is to check whether the following ratios defined by

$$
\left\{\begin{array}{l}
r_{\phi}=\frac{K \sigma_{\phi} d_{\phi}}{\sigma_{\text {image }}} \quad \text { for phase and } \\
r_{\phi^{\prime}}=\frac{K \sigma_{\phi^{\prime}} d_{\phi^{\prime}}^{2}}{\sigma_{\text {image }}} \quad \text { for phase derivative }
\end{array}\right.
$$

are constant. These constant values are expected to be equal to

$$
\left\{\begin{array}{l}
r_{\phi}=\frac{3}{\sqrt{2 \pi}} \times \sqrt{\Delta_{x} \Delta_{y}} \simeq 1.1968 \text { pixel } \\
r_{\phi^{\prime}}=\frac{9}{\sqrt{\pi}} \times \sqrt{\Delta_{x} \Delta_{y}} \simeq 5.0777 \text { pixel }
\end{array}\right.
$$

according to Equations 9 in which $\Delta_{x}$ and $\Delta_{y}$ are both equal here to one (size of a pixel given in pixel). Since these equations involve several standard deviations, namely $\sigma_{\phi}, \sigma_{\phi^{\prime}}$ and $\sigma_{\text {image }}$, checking that they are actually satisfied needs to work with a set of grid images, each of them being corrupted by a different copy of noise whose standard deviation is $\sigma_{\text {image }}$. Two phase and four phase derivative maps are deduced from each of these grid images, and one can then determine the empirical standard deviation of each of these quantities at each pixel too.

In practice, a set of $2001000 \times 1000$ synthetic images has been generated using the model described in Equation 1. The following rules are adopted:

- The grey level $s$ is quantised on 16 bits to mimic the 16-bit quantisation of recent cameras;

- The amplitude of the carrier $\left(\frac{A}{2}\right)$, which directly governs the amplitude of $K$, lies between $2^{12}$ and $2^{14}$. It linearly increases along the horizontal axis;

- The contrast $\gamma$ is equal to one;

- The frng function which drives the profile of the grid is a sine function;

- The number of pixels per grid period is equal to five;

- A Gaussian white noise $n$, whose standard deviation $\sigma_{\text {image }}$ lies between $2^{7}$ and $2^{10}$, is added to the simulated grid image. $\sigma_{\text {image }}$ linearly decreases along the vertical axis of the synthetic grid images, from the top to the bottom;

- The imposed displacement field is rigorously null to focus here only on noise, so the phase of the carrier is not modulated. 
A typical simulated grid is shown in Figure 1. Small squares extracted near the four corners are reported in Figure 2. Their exact location on the simulated grid is shown in Figure 1 where four small white squares have been superimposed. It can be seen that contrast and brightness increase from the left to the right. Additive noise significantly corrupts the grid image at the bottom left. Noise effect is not so clearly visible at the bottom right because the contrast is much higher here.

Since the effect of high brightness and low noise are combined at the top right of the simulated grid, it is expected to find the lowest standard deviation, thus the best resolution, in this zone for both the phase and the phase derivative. On the contrary, the zone located at the bottom left is penalised by low brightness and high noise level, so it is expected to find the highest standard deviation, thus the worst resolution, in this zone for both the phase and phase derivative.

200 grid images have been generated using the rules defined above with independent copies of noise, so it makes sense to calculate the empirical standard deviations of the phases and phase derivatives at any pixel to characterise their temporal distributions from one image to another. The obtained results being similar for the phases along $x$ and $y$ on the one hand, and for the four resulting phase derivatives on the other hand, only $\Phi_{x}$ and $\frac{\partial \Phi_{x}}{\partial x}$ serve as an illustration here.

Figure 3-a shows the distribution of the standard deviation of the phase $\sigma_{\phi}$ at the pixels of this set of 200 phase maps. As expected, $\sigma_{\phi}$ is maximum at the bottom left and minimum at the top right.

Figure 4-a shows the $\sigma_{\phi^{\prime}}$ distribution. The same remark as above holds. It can also be noted that derivation induces the shape of the "blobs" in the map to become somewhat directional while they are isotropic for the phase in Figure 3-a. It can be checked on the other phase derivatives maps (not reported here) that this direction rotates by 90 degrees when differentiation is performed with respect to $y$ instead of $x$. This conforms to the theory developed in [36].

The ratios $r_{\phi}$ and $r_{\phi^{\prime}}$ which are defined in Equations 12 can be calculated with the 200 phase maps and 200 phase derivative maps, at each of their pixels. This provides the maps shown in Figures 3-b and 4-b.

The most striking point is that no difference in both the shape and the amplitude of the blobs characterizing noise can be observed from one region of these maps to each other whereas this was the case for the maps of the phase and phase derivative standard deviations. This lends credence to the fact that we obtain constant values for $r_{\phi}$ and $r_{\phi^{\prime}}$ whatever the value of $K$ and $\sigma_{\text {image. }}$ However, since we deal here with random distributions, this property can only be deemed as statistically significant. The mean value of $\frac{K \sigma_{\phi} d_{\phi}}{\sigma_{\text {image }}}$ is equal to 1.1921 pixel and the mean value of $\frac{K \sigma_{\phi^{\prime}} d_{\phi^{\prime}}^{2}}{\sigma_{\text {image }}}$ to 5.0599 pixel. These mean values are very close to their theoretical counterparts given in Equation 12 above: 1.1968 pixel and 5.0777 pixel, respectively, thus validating Equations 6 . 
These distributions around the theoretical values can be more clearly seen with the histograms shown Figures 3-c and 4-c where $r_{\phi}$ and $r_{\phi^{\prime}}$ have been normalised by dividing them by their theoretical values given in Equation 12 above: $\frac{3}{\sqrt{2 \pi}} \simeq 1.1968$ pixel and $\frac{9}{\sqrt{\pi}} \simeq 5.0777$ pixel, respectively, thus $r_{\phi}^{\text {norm }}=\frac{\sqrt{2 \pi}}{3} \times r_{\phi}$ and $r_{\phi \prime}^{\text {norm }}=\frac{\sqrt{\pi}}{9} \times r_{\phi \prime}$. Both histograms are perfectly symmetric with respect to 1 and only slightly scattered since the standard deviation is 0.0495 for $r_{\phi}$ and 0.0500 for $r_{\phi^{\prime}}$. Student's t-test shows that, from these data, the p-values associated to the hypotheses " $r_{\phi}$ is 1.1968 pixel" and " $r_{\phi^{\prime}}$ is 5.0777 pixel" are respectively 0.26 and 0.32 , thus greater than a significance level of 0.05 . The relationships are thus statistically significant [9].

\subsection{Trade-off between resolution and spatial resolution}

The above simulations were performed with a constant value of the kernel size $\sigma$, so for a fixed spatial resolution $d_{\phi}=d_{\phi^{\prime}}$. We consider now a new grid image obtained with a constant value of $\sigma_{\text {image }}$ and with a constant carrier amplitude $A$ and contrast $\gamma$, so $K$ is approximately constant throughout the image. It must be pointed out that it is not rigorously constant since it slightly changes from one pixel to each other, depending on the grey level of the pixel at which the Fourier transform is calculated, as demonstrated in $[36]$.

Equations 9 tell us that for given settings of the camera employed to capture the grid images, there is competition between resolution and spatial resolution: the greater the former, the lower the latter. As discussed in Section $3, \sigma_{\phi}$ and $\sigma_{\phi^{\prime}}$ change in a different way. The following simulation illustrates this result. The phase carrier remains here unmodulated to focus only on the effect of noise.

The noise in phase and phase derivative maps has been characterised for various values of $\sigma$ by calculating the empirical estimation of the standard deviations $\sigma_{\phi}$ and $\sigma_{\phi^{\prime}}$, and comparing the obtained results with those predicted with Equations 8 . The comparison between predicted and empirical quantities as well as the influence of $\sigma$ (and therefore of the spatial resolution) can be seen in Figure 5, where $\phi_{x}$ and $\frac{\partial \phi_{x}}{\partial x}$ have been considered. Very similar results have been obtained for the other phase (along $y$ instead of along $x$ ) and phase derivatives, so they are not reported here.

It is clear that the observed standard deviations are nearly the same as the predicted ones. It is worth mentioning that $\sigma_{\phi}$ is inversely proportional to $d_{\Phi}$ while $\sigma_{\phi^{\prime}}$ is inversely proportional to the square of $d_{\Phi^{\prime}}$, so $\sigma_{\phi^{\prime}}$ more quickly decreases than $\sigma_{\phi^{\prime}}$. This can potentially be very useful to filter noise in strain maps, but actual details would also be impaired at the same time. Further work should be undertaken to find a trade-off, also keeping in mind that deconvolution of strain maps is possible if noise is thoroughly characterised [16]. 


\section{Experiments: first results}

\section{$5.1 \quad$ Introduction}

The objective here is to consider a real practical case and to check whether the predictive equations discussed above on synthetic data still remain valid. The main differences with the simulations above are threefold:

1. First, the actual marking is not so regular as the simulated one. For instance, slight changes in the grid pitch are observed, as discussed in [5], and the WFT detects such changes which appear in the phase derivative maps as parasitic waves perpendicular to the direction of derivation. As explained above, this is the reason for which temporal fluctuation of the phase and phase derivative at each pixel of a series of shots are considered rather their spatial changes;

2. Since repeated shots of the same scene are collected to detect and quantify temporal fluctuations of the grey level at each pixel, images are potentially disturbed by other phenomena than noise of the camera which can occur at the same time, for instance light flicker, micro-oscillations of the deck on which both the camera and the tensile machine where the specimen is fixed, or slight non-constant control of the grip of the tensile machine where the specimen is fixed. Such phenomena are not visible to the naked eye but are potentially detectable by the camera, as illustrated below;

3. Assuming a homoscedastic noise as in the simulations presented above is convenient and widely used for general purpose in image processing. This assumption is known to be flawed for raw data provided by digital imaging sensors [12]. A more adequate modelling is to consider noise variance as a linear function of the grey level, as it is often mentioned in the data-sheets of camera suppliers. This peculiar property has also been observed here.

This section is organised as follows. The experimental setup is first briefly described. A mean value of the standard deviation in a set of 200 grid images is then estimated to check if Equations 10 are satisfied. Since this is not the case, a thorough investigation of noise is carried out and a relevant heteroscedastic model is proposed and identified. A dedicated variance stabilisation procedure is then applied to correct the grid images and it is observed that Equations 9 and 10 are eventually satisfied.

\subsection{Experimental setup}

A bidirectional grid featuring a theoretical pitch of $0.2 \mathrm{~mm}$ was transferred using the procedure described in [28]. The transferred grid features some localised defects due to lack of ink. It also has slight spatial pitch fluctuation due to printing, as already 
discussed in Section 3 above. The dimensions of the grid employed here to characterise noise were limited to $24 \times 40 \mathrm{~mm}^{2}$ because of some issues with computer memory due to the large amount of images to be processed to perform a statistical analysis. Since 5 pixels were used to encode one grid pitch, this lead to grid images whose dimensions are $600 \times 1000$ pixels. Note that a strip of 50 pixels has been removed around the edge of some maps of results shown in the paper to avoid outliers due to edge effects, so the dimensions of the maps are $500 \times 900$ pixels in these cases.

A SENSICAM QE camera featuring a 12-bit/1040×1376-pixel sensor and equipped with a $105 \mathrm{~mm}$ SIGMA lens was used to capture the images of the grid. Note that the grey level of the images provided by this camera is digitised at 16 bits and not at 12 , so the jump between two consecutive grey levels is equal to $2^{4}=16$. The distance between camera and specimen was adjusted in such a way that 5 pixels were employed to encode one grid pitch. The location of the camera was thoroughly adjusted so that the lines of the grid were parallel to the pixels of the sensor along both the horizontal and vertical axes of the reference configuration.

During each test, the grid was illuminated by three flexible and movable light guides fed by a KL 2500 LCD cold light source. The location of the light guides was adjusted so that the lighting of the grid was nearly uniform to the naked eye.

The grid was fixed in the moving grip of a testing machine, the other grip remaining open so that no tensile/compression force was applied to the specimen. This enabled us to take images of the grid in a given position to investigate first noise propagation in phase and phase derivative maps, and then to translate the specimen by $0.1 \mathrm{~mm}$, take a new set of grid images and study noise propagation in displacement and strain maps. The former quantity is expected to be theoretically constant and thus the latter null. 200 images of this grid were shot for each position of the grip. A picture showing a very similar experimental setup as that used in the present study is shown in [17].

\subsection{Estimation of the displacement and strain resolutions with an equivalent standard deviation of noise in the whole set of grid images}

The objective here is to check whether Equations 10 are satisfied with a simplistic homoscedastic noise assumption (phase and phase derivations are not considered here but they will be examined in the following sections). Let us assume for a while that noise has a constant variance across the image. A single value for the standard deviation of noise in the grid images $\sigma_{\text {image }}$ must be estimated for this purpose. This value can be obtained first by calculating this standard deviation pixel by pixel. An equivalent and global value denoted $\sigma_{\text {image }}^{\text {equivalent }}$ is then deduced. It is defined by 


$$
\sigma_{\text {image }}^{\text {equivalent }}=\sqrt{\frac{1}{N} \sum_{i=1}^{N} \sigma_{\text {image }}^{2}\left(x_{i}, y_{i}\right)}
$$

where $N$ is the number of pixels in the grid image. Considering the sets of 200 grid images, $\sigma_{\text {image }}^{\text {equivalent }}$ is equal to 407 grey levels for the reference location of the grid and 441 grey levels after translation. It means that this quantity depends on the location of the grid and/or on lighting conditions and/or on other image acquiring conditions. We

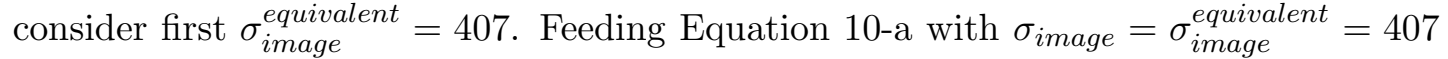
enables us to deduce $\sigma_{u}, d_{u}$ being determined by the size of the kernel in the WFT equal to $\sigma=5$ pixels here, thus $d_{u}=30$ pixels. The apparent displacement resolution for the vertical displacement deduced from the 200 grid images is shown in Figure 6-a. This map should be compared with the map of the displacement resolution predicted using the empirical procedure described above. This map is shown in Figure 6-b. As may be seen, these maps are totally different. This is confirmed by the histogram of the ratio between these two maps shown in Figure 6-c. It can be seen that the distribution is not Gaussian and that the apparent resolution is nearly 6 times greater than the predicted one, meaning that the procedure described above does not work for displacement.

The same procedure applied to the strain leads to the maps shown in Figure 7. This time however, the maps in Figure $7-\mathrm{a}$ and $-\mathrm{b}$ exhibit the same trends but the estimated distribution is greater than the measured one. This is confirmed by the ratio between both quantities shown in Figure 7 -c. The mean ratio is equal to 0.87, which means that a mean error equal to $13 \%$ is obtained when using the procedure described above, which can be considered as quite acceptable. Note however that using 441 pixels instead of 407 for $\sigma_{\text {image }}^{\text {equivalent }}$ leads to a greater error, namely $20 \%$ instead of $13 \%$, but the order of magnitude between measured and estimated resolutions remain the same. Note finally that the spatial distribution of this ratio (not shown here) shows that it depends on $K$ (thus, roughly speaking, on brightness): the higher the value of $K$, the smaller the error.

The fact that the predictive equation is not satisfied at all for the displacement map and only roughly satisfied for the strain map means that the assumptions under which these equations are obtained are not verified, especially concerning the noise homoscedasticity assumption. It is therefore necessary to investigate in more detail this issue. This is the objective of the following sections.

\subsection{First observation: heteroscedastic noise}

Let us now consider in more detail the spatial noise distribution over the grid. Variances are considered here instead of standard deviations, as justified by the calculations carried out in Section 6 below. Considering this distribution leads to an important result in the context of the simulations performed above since variance changes from one pixel 
to each other. More precisely, variance is all the more significant as the grey level of the pixel under consideration is high. It means that noise is actually not homoscedastic but heteroscedastic. Since we have a series of 200 grid images, it is possible to compute the average grey level and the empirical variance. Homoscedasticity can be observed in Figure 8-b where a close-up view of the variance distribution is shown. A close-up view of the average grid is shown in Figure 8-a for comparison purposes. This zone is defined by $100 \geq x \geq 50$ and $100 \geq y \geq 50$ in the grid images. The grid pattern is clearly recognizable in Figure 8-b. The reason is that the variance is small along the black lines of the grid and high at the bright spots bordered by the lines. Consequently, the spatial distribution of the variance has exactly the same pattern as that of the grid. The grey-level dependency of the variance is illustrated in Figure 9-a which represents the variance vs. the grey level at all the pixels of the grid image. This point cloud exhibits a very clear linearly increasing trend. This trend is confirmed by the data-sheet of the Sensicam camera supplier which claims that variance evolves at a linear rate with respect to grey level [1]. It will be seen in Section 6 below that this heteroscedastic noise can be changed into a homoscedastic one but this change is corrupted by another phenomenon illustrated by the upper border of the point cloud which seems to be more irregular than the lower one. This feature means that an additional phenomenon occurs, as illustrated in the following section.

\subsection{Second observation: micro-movement}

Figure 10 shows the grey level vs. time at different types of pixels: at the center of the crossing between two dark lines, at a point located at the top of a horizontal line and between two crossings, at the bottom of the same horizontal line, and at the center of a bright spot (see plain lines in the figure). A random evolution is first visible. On close inspection however, another slight phenomenon also appears: a low-frequency variation of the local temporal mean value of this random evolution. It is most visible for the curves plotted at the bottom of a line and at the top of a line. It can be seen that these lines seem to slightly move outward nearly between images 30 and 60 , then between images 130 and 160. They move inward between these two time periods. The physical reason of this phenomenon is as follows: these out-of-phase fluctuations correspond to a vertical micro-movement of the camera with respect to the grid. Despite the camera having been carefully fixed on a heavy tripod, camera shake seems still noticeable at the pixel scale. This micro-movement due to micro-oscillations is confirmed by the fact that the lines where the vertical grey-level gradient is the highest are the most sensitive to this phenomenon, contrary to the lines corresponding to the maximum and minimum grey level where this low-frequency fluctuation is the less visible (compare the four curves in Figure 10). This effect is quite subtle but it leads the apparent grey level variance in the grid images to result from both the noise in camera sensor and this micro-movement. Note finally that this problem would certainly be avoided or at least 
significantly reduced by performing the experiments on a vibration-isolating optical table. It is however important to show in this paper that performing such experiments under usual laboratory conditions gives rise to these undesirable vibrations, but that their negative effect can be removed with appropriate image processing.

\subsection{Removing the non-random part of the signal and estimating the noise variance}

An appealing idea when considering digital noise characterisation would be to use time averaging from a series of static images to get consistent estimators of both the average grey level and the associated noise variance. However, micro-movements make it impossible to estimate noise variance at a given pixel $(x, y)$ by this method without any pre-processing, since the temporal variation of the value of the grey-value of a given pixel along time is in this case not solely due to sensor noise, but also to microoscillations of the specimen with respect to the camera. It is interesting to note that dedicated studies take advantage of such altered images to characterise vibrations [29, 30,27 . It turns out here that in the case of grid images, it is possible to remove these non-random variations by using the particular periodical nature of the grid. This procedure, called non-random signal reduction (NRSR) in the remainder of the paper, is described in details in [35]. For each grid image, it consists in performing a spatial average of the grey values over pixels distant from multiple of the grid pitch around any given pixel of the grid raw image to get the noise-free component of the signal. The result is then subtracted from the raw grid images in order to get the actual sensor noise component. In practice, the average is computed with Gaussian weights with respect to the distance from the considered pixel, as justified in [35].

This effect of the NRSR is illustrated in Figure 11 which shows the grey level evolution vs. time of the top and bottom of a typical horizontal line. The evolution before NRSR is recalled first in Figure 11-a for comparison purposes. Removing the highest frequencies (thus sensor noise) by applying the procedure explained in Ref. [35] gives the curve depicted in Figure 11-b, where the out-of-phase fluctuation due to microvibration is clearly visible. Removing now this fluctuation from the original signal gives the curve shown in Figure 11-c which is considered as the signal provided by the camera without the influence of micro-oscillations.

The effect of this NRSR on this curves is quite subtle but it strongly affects the calculation of the variance of the grey level evolution at any pixel. This can be seen by plotting in Figure 9-b the same type of point cloud as in Figure 9-a, but after applying the NRSR. It can be seen that the point cloud is now narrower and much more regular than without this correction. This type of distribution (apart from the outliers which correspond to the pixels where black ink is missing) is now in agreement with the linear distribution claimed by the data-sheet of the camera [1]. 


\subsection{Estimation of the displacement and strain resolutions with an equivalent standard deviation of noise after NRSR}

While Section 5.6 shows that a part of the heteroscedasticity of the noise can be explained by micro-movements, one can wonder if, after having removed the effects of these micro-movements, it would be a sound simplification to still model the noise as a homoscedastic process. The approach proposed in Section 5.3 above, in which an equivalent standard deviation is introduced, is reconsidered for this purpose but after applying the NRSR on the set of 200 grid images. The equivalent standard deviation is estimated again using Equation 13. In this case, $\sigma_{\text {image }}^{\text {equivalent }}$ is equal to 352 grey levels for the reference location of the grid and 354 grey levels after translation, instead of respectively 407 and 441 in Section 5.3. It is clear that the standard deviation is lower after NRSR, which is quite logical since the influence of micro-vibration is expected to be removed after NRSR. Interestingly, the standard deviation is now very close in both sets of images, which indicates that the influence of the micro-movement has been actually eliminated, and that a more reliable estimation of the global standard deviation of the noise in the grid images has been obtained.

Identifying first the displacement and strain resolutions from the 200 grid images for which the NRSR has been applied gives the maps shown in Figures 12-a and Figures 13-a. Compared to Figures 6-a and 7-a, it can be observed that the resolution in displacement has significantly changed while that in strain remains nearly unchanged. This is due to the fact that the micro-movement is rigid-body like, thus it only affects displacements and not strain components. Consequently, the NRSR influences only the first quantity, not the second one.

Feeding now Equations 10-a and -b with $\sigma=\sigma_{\text {image }}^{\text {equivalent }}=352$ to try to predict the displacement and strain resolutions $\sigma_{u}$ and $\sigma_{\epsilon}$ gives two maps directly proportional to those already shown in Figures $6-\mathrm{b}$ and 7 -b. They are therefore not shown here (the colorbars in Figures 6-b and 7-b must merely be multiplied by 352/407). These predicted distributions seem to be very similar to the identified resolution maps shown in Figures 12-a and 13-a. This is illustrated by the histogram of the ratio between identified and estimated resolution shown in Figures 12-b and 13-b. The first histogram is centred on 0.96 , the second one on 0.99 , showing that the average values of the estimated displacement and strain resolutions are much better than without NRSR. They are actually quite satisfactory on average. However, plotting the spatial distribution of this ratio (see Figures 12-c and 13-c) shows that that these ratios are not randomly distributed: they actually depend on the brightness distribution over the specimen. This brightness features some significant local deviation, as discussed below. This illustrates that estimating displacement and strain resolutions with this approach is too rough, certainly because Equations 10 are obtained assuming noise is homoscedastic while it is actually heteroscedastic. This is an issue for further use of noise characteristics in displacement or strain maps, for instance for deconvolution purposes [16]. A more 
sophisticated approach must therefore be employed to correctly tackle the problem of heteroscedastic noise. This is the objective of the next section.

\section{Estimation of the displacement and strain resolutions after noise normalisation in the grid images with the generalised Anscombe transform}

\subsection{Principle}

Grid images are impaired by noise, which is inherent to any digital signal acquisition device. In the considered experimental setting, the camera response function is linear. It means that, disregarding the analog-to-digital conversion, the grey level measured at a pixel is proportional to the quantity of light photons arriving at a CCD sensor cell, plus a dark current [20]. The following simplified yet realistic stochastic model is often assumed $[8,12,14]$ :

$$
s(x, y)=g \cdot \eta_{p(x, y)}(x, y)+\delta(x, y)
$$

where:

- $s$ is the observed signal;

- $g>0$ is the gain of the electronic system;

- The number of collected light photons $\eta_{p(x, y)}(x, y)$ at a pixel $(x, y)$ is a random variable following a Poisson distribution of parameter $p(x, y)$, assumed to be spatially independently distributed;

- The dark current $\delta(x, y)$ is a Gaussian white noise of mean $\mu_{d}$ and variance $\sigma_{d}^{2}$. It is present, yet less strong, even if a cooling system is used as in the Sensicam camera employed in the present work.

The random variables $\eta_{p(x, y)}(x, y)$ and $\delta(x, y)$ are assumed to be independent. With these assumptions, it is possible to derive:

$$
E(s(x, y))=g p(x, y)+\mu_{d}
$$

and

$$
\operatorname{Var}(s(x, y))=g^{2} p(x, y)+\sigma_{d}^{2}
$$

where $E$ and Var denotes respectively the expectation and the variance of a random variable.

As in $[8,14,31]$, the following linear relation between the expectation and the variance of $s(x, y)$ holds (from Equations 15 and 16):

$$
\operatorname{Var}(s(x, y))=g E(s(x, y))+\sigma_{d}^{2}-g \mu_{d}
$$


As noted in [14], it is possible that $\sigma_{d}^{2}-g \mu_{d}<0$.

The noisy signal is thus a heteroscedastic random variable, and its variance linearly depends on its expectation. However, most denoising algorithms assume homoscedasticity. A popular workaround is to stabilise first the heteroscedastic process. The Anscombe transform [3] is an efficient tool to reach this goal. It maps a random variable with a Poisson distribution into a random variable with an approximately standard Gaussian distribution. A generalisation of this approach, namely the generalised Anscombe Transform (GAT), has been proposed in [26] to stabilise to 1 the variance of a Poisson-Gaussian variable as the one in Equation 14. With the above-mentioned notations, the GAT writes as follows:

$$
\operatorname{GAT}(s(x, y))=\frac{2}{g} \sqrt{g s(x, y)+\frac{3}{8} g^{2}+\sigma_{d}^{2}-g \mu_{d}}
$$

\subsection{Application to the grid images}

The coefficients involved in the GAT defined in Equation 18 can be easily identified from the parameters of the linear relation between the variance and the expectation of the grey level values given in Equation 14. The objective is now to estimate these coefficients in the present example to modify the grey levels of the grid image already discussed above. The technique employed here was first discussed in [7] and further promoted in $[8,11,31]$.

A robust least square estimate first gives the coefficients of the straight line defined by Equation 17 which fits the point cloud shown in Figure 9-b: $g=8.319$ and $\sigma_{d}^{2}-$ $g \mu_{d}=-5239$. It can be noted that the constant coefficient is slightly negative here, as discussed in [14] for instance. Note that these coefficients can be estimated from a single image [14], permitting to get rid of the micro-movement problem. However the algorithm described in [14] requires a much complex segmentation step to get level sets than the simple procedure described in the present article. A similar procedure to that described in Ref. [14] would be to use the periodicity of a single grid image to define the level sets (whose grey-level variation is explained only by noise). This has not been investigated further here. Note also that a procedure to characterise the noise by time-averaging is discussed in [13], but camera shake is overlooked in this article.

Calculating then the GAT of the images and deducing the variance at any pixel gives the point cloud shown in Figure 9-c. The variance is now remarkably approximately equal to one whatever the brightness, thus illustrating the effect of normalisation performed by the generalised Anscombe transform. Consequently the $\operatorname{GAT}(s(x, y))$ are actually homoscedastically distributed with a variance of 1 , while the $s(x, y)$ are heteroscedastically distributed. Note finally that the grey level in the images being impacted by the generalised Anscombe transform, the scale along the horizontal axis is different in Figure 9-c and in Figures 9-a-b. 
The impact of both the NRSR and the GAT on the standard deviation of the noise in the grid images can be visually assessed in Figures 8-c and -d. Applying the GAT on the raw grid images without any NRSR leads to Figure 8-c. The normalised standard deviation is close to one but the grid pattern is still visible. It means that noise is not yet really homoscedastic. Applying the NRSR prior to the GAT actually stabilises the variance since the grid pattern is no more visible in the variance map. In addition, the variance is now close to one (see Figure 8-d), thus illustrating that noise is now normalised and homoscedastic.

As a last remark, it must be emphasized that the GAT has an impact only on the grey level of the grid images but the phases and their derivatives remain theoretically the same, see [36]. It means that only the nature of the noise in the final phase and phase derivative maps will theoretically be affected (and not the phase and phase derivative themselves). It is therefore possible to check whether Equations 9 are satisfied since we deal here with homoscedastic noise in the images, thus whether the resolution can be predicted in the phase and the phase derivative maps. This is the objective of the next section. It is noteworthy that no inverse GAT [23] is needed in the present work, contrary to most image processing applications (see, e.g., [8]).

\subsection{Predicting the resolution in the phase and phase derivative maps}

The objective is now to process normalised grid images in order to extract the phase and phase derivatives for the 200 grid images discussed above. We focus here only on these quantities calculated along direction y. This is the vertical direction along which the translation is performed, so the largest errors in the phase and phase derivative maps are expected to be obtained in this case. $\sigma=5$ pixels is chosen for the standard deviation of the Gaussian window of the WFT. The spatial resolution $\left(d_{\phi}=d_{\phi^{\prime}}\right.$ in this study, even though the phase and its derivatives can be found independently [5]) is therefore equal to $6 \times 5=30$ pixels.

The standard deviation, thus the resolution as defined in Section 1, can easily be calculated at any pixel for the phase and the phase derivative. These resolutions (one for the phase, one for the phase derivative) will then be compared to the resolutions predicted with Equations 9 where $\sigma_{\text {image }}=1$ since noise is now normalised with the Anscombe transform, and $\Delta_{x}=\Delta_{y}=1$ since dimensions are directly in pixels. $K$ can be the average value for the modulus of the WFT calculated for the 200 normalised grid images, but since it only very slightly changes from one grid image to each other, the modulus of the WFT of the last grid image of the series can be considered in the calculations for convenience. We use here the value of $K$ calculated for the normalised grid images but the modulus obtained with the raw grid images is also shown for didactic purposes.

The maps showing the spatial distribution of the mean of $K$ for both the raw grid images and the normalised (NRSR+GAT) are shown in Figure 14. As predicted by 
equation $7, K$ is proportional to the illumination $A$ which gently varies across the grid image. Normalisation has an impact on the grey level, thus on the modulus $K$. In Figure 14, the scales are therefore different for the normalised and the raw grid images. Despite this difference, it can be checked that the normalised modulus is smoother than the initial modulus. This is due to the very definition of the GAT which involves a square root of a linear expression of the grey level.

Figure 15 shows the mean phase distribution for both the initial and the normalised grid images (NRSR+GAT). No real difference can be observed to the naked eye between Figures 15-a and Figures 15-b, thus illustrating that the GAT has practically no impact on these quantities. On close inspection however, a slight difference is observed. In this example, it is lower than $1 \%$. Note also that the phase (either normalised or not) is not constant while the specimen does not deform. This is due to a slight difference between the theoretical number of pixels per period used to encode the grid image ( 5 in this case) and that obtained during the test. It causes a small isotropic strain to appear and to modulate the phase distributions in both the reference and the current phase maps. Since displacement maps are obtained by subtracting current and initial phase maps, this initial phase distribution has no impact on displacement maps.

Figure 16 now shows the phase derivative maps along the vertical direction $(y)$. This phase derivative is calculated using the procedure described in [5]. It relies on a convolution of the grid picture with a suitable kernel. The advantage is to directly provide phase derivative maps, thus avoiding issues due to phase jumps when differentiating phase maps where such jumps occur. A regular pattern made of horizontal lines clearly appears in Figure 16. It is due to very slight fluctuations of the grid pitch caused by printing, as discussed in [5]. These fluctuations are tiny: some micrometers at most but they induce parasitic phase changes which are magnified by differentiation. Most of these parasitic fringes are removed in the final strain maps by projecting the phase derivative map in the coordinate system of the reference images prior to subtracting current and reference phase derivative maps, as explained in [5]. Figure 16-c shows that the error due to the GAT is magnified by differentiation. It can be checked that it remains however smaller than about 5\%. This phenomenon has not been investigated in the present study, but further work should be undertaken to see how to remove or limit its negative effect. It has no real impact on the prediction of the metrological performance which are based on the fluctuation of the phase and phase derivative, and not on these quantities themselves.

The standard deviation, thus the resolution at any pixel of the phase and phase derivative maps has been calculated from the 200 grid images obtained after NRSR and GAT. The maps deduced from these grid images are shown in Figures 17-a and 18-a for the phase and phase derivative, respectively. Comparing these maps with those of the WFT modulus $K$ clearly illustrates that from a qualitative point of view, the higher the modulus, the lower the resolutions for the phase and its derivative, as predicted by Equations 9. A black circle is superposed at the top left of both maps. 
Its diameter is equal to the spatial resolution, thus to the width of the Gaussian kernel equal to $6 \times \sigma=30$ pixels. This type of figure enables us to see at a glance the metrological performance of the technique for retrieving the phase and its derivative: the circle provides the spatial resolution which is constant throughout the map while the resolution changes from one point to each other because $K$ changes throughout the grid images.

Let us now compare these measured quantities with those predicted with Equations 9 in which $\sigma$ is now

- Option 1: close to one by considering the variance of the grid image obtained when the GAT is applied; or

- Option 2: close to one directly by enforcing the variance to be equal to one.

$K$ is the modulus of the WFT calculated for the normalised grid images. This modulus is denoted $K_{\text {norm }}$. Equations 9 reduce in this case to these very simple expressions

$$
\begin{cases}\sigma_{\phi} \times d_{\phi} & =\frac{3}{\sqrt{2 \pi} K_{\text {norm }}} \quad \text { for phase and } \\ \sigma_{\phi^{\prime}} \times d_{\phi^{\prime}}^{2} & =\frac{9}{\sqrt{\pi} K_{\text {norm }}} \quad \text { for phase derivative }\end{cases}
$$

The predicted spatial distributions are shown in Figure 17-b for the phase and in Figure 18-b for the phase derivative. These standard deviations are calculated here pixelwise with the actual variance obtained after applying the GAT to the grid images (option 1 above). No noise correlation is taken into account, so the corresponding distributions are not as smooth as the measured one. One can however see that both the amplitude and the trends for the spatial distributions are very similar for both predicted and measured resolution.

Finally, enforcing the variance to be equal to one (option 2 above) gives the map shown in Figure 17-c for the phase and in Figure 18-c for the phase derivative. Again, predicted and measured distributions are very similar, thus validating Equations 19.

\subsection{Resolution in displacement and strain}

Similar results for displacement and strain can be obtained by using Equations 10 above if normalised grid images are used. $\sigma_{\text {image }}$ is therefore equal to one and $K$ is the modulus of the WFT of the normalised grid images calculated for the reference grid image, thus $K=K_{\text {norm }}$. Equations 10 reduce in this case to 


$$
\left\{\begin{aligned}
\sigma_{u} \times d_{u} & =\frac{3}{2 \pi^{3 / 2}} \times \frac{p}{K_{\text {norm }}} \quad \text { for displacement and } \\
\sigma_{\epsilon} \times d_{\epsilon}^{2} & =\frac{9 \sqrt{2}}{2 \pi^{3 / 2}} \times \frac{p}{K_{\text {norm }}} \quad \text { for strain. }
\end{aligned}\right.
$$

The distributions of the resolutions $\sigma_{u}$ and $\sigma_{\epsilon}$, which are deduced from the 200 phase and phase derivative maps in the reference and current locations, are shown in Figures 19-a and 20-a for the displacement and strain, respectively. Normalised grid images are considered in this case. They are obtained by taking the grid image $i, i=1 \cdots 200$, obtained when no displacement is imposed, and image $i$ when a vertical displacement equal to $0.1 \mathrm{~mm}$ is applied. The predicted distributions of $\sigma_{u}$ and $\sigma_{\epsilon}$ obtained with Equations 20 are shown in Figures 19-b and 20-b for the displacement and the strain, respectively.

It can be seen that experimental and predicted maps are very similar. The main difference is due to the fact that the predicted maps do not take into account noise correlation characterised in [36]. The histograms of the distribution of the ratio between measured and predicted resolutions are shown in Figures 19-c and Figures 20-c for the displacement and the strain, respectively. Both distributions are sharply distributed around 1 . The actual mean value is equal to 0.961 for the displacement and 0.993 for the strain.

It is also interesting to compare the preceding results with those obtained with the raw grid images already discussed in Section 5.3 and shown in Figure 6-a for the displacement and Figure 7-a for the strain. Comparing Figures 19-a and Figure 6a shows that the resolution maps obtained with raw and normalised grid images are completely different. In particular, the resolution is worse with raw images than with normalised ones. This can be explained by the micro-movement that corrupts image acquisition and biases the estimation of the variance, as discussed in Section 5. This phenomenon is illustrated in Figure 21, which shows the vertical displacement (along the $y$-direction) at three pixels of the displacement map vs. time. The measured vertical displacement is close to 2.5 pixel This quantity corresponds to the $2.5 \times 40=$ 100 micrometers prescribed by the moving grip of the testing machine. The three curves are superimposed. This confirms that the specimen undergoes a rigid-body-like micromovement, more precisely a very small vertical translation, as discussed in Section 5.5. The peak-to-peak amplitude of this micro-movement is equal to about 0.1 pixel, thus about 4 micrometers. The fact that such a small movement significantly disturbs the apparent noise variance in the grid images, as discussed in Section 5.5 above, underlines the sensitivity of the grid method for small displacement measurement.

Comparing now Figures 20-a and Figure 7-a shows that the resolutions obtained with raw and normalised images are very similar. This is quite logical since the micro- 
movement is actually a translation which only affects displacement maps and not strain maps. The histogram of the ratio between these two quantities is shown in Figure 22a. The mean value for this ratio is equal to 1.048. Since it is shown above that the predicted resolution obtained with normalised images is very close to the identified resolution, it means that Equation 20-b provides a reliable estimate of the resolution for the strain obtained with raw grid images, not only with normalised grid images.

A last histogram in Figure 22-b shows the ratio between the distributions of the resolution estimated with raw and normalised grid images. It can be seen that the distribution is no longer Gaussian. It exhibits two peaks: one for the dark lines for which the resolution is low since $\sigma_{\text {image }}$ is small in this case, and another one for the bright spots for which $\sigma_{\text {image }}$ is high. It is also clear that the distribution is very large in this case, thus highlighting the benefit of the procedure consisting in applying the NRSR and the GAT prior to estimating the resolution for the set of 200 images.

Using the GAT after the NRSR was justified by the fact that the resolutions predicted by using an equivalent standard deviation in Equations 10 lead to correct values on average, but to spatial distributions of these values which are sensitive to brightness, as discussed at the end of Section 5. To clearly see the benefit of the GAT in addition to applying the NRSR, the spatial distributions of the ratios whose histogram are shown in Figures 19-c and 20-c, are shown in Figure 23. As may be seen, these ratios do not depend on lighting intensity anymore since they are now randomly spatially distributed around 1. This was not the case after NRSR only, as illustrated in Figures 12-c and 13-c. This allows further use of this a priori estimation of the resolutions for deconvolution purposes for instance [16].

\section{Conclusion}

This paper shows that it is possible to predict the resolution in displacement and strain maps obtained with the grid method, provided that the noise process that impairs the grid images is correctly characterised. This contribution to the noise floor in these maps only takes into account the noise due to the camera, not spatial fluctuations due to other phenomena such as grid defects. These predictions are obtained with suitable expressions which clearly show that the metrological performance is all the more satisfactory as brightness and contrast are high. These quantities being in general not uniform on the gauge section of the tested specimens, it means that resolution is not constant throughout displacement and strain maps.

These closed-form equations for resolution prediction were first successfully checked with numerical simulations. Addressing real grid images was more challenging for two main reasons. First it has been observed that micro-movements corrupt grid images, thus a dedicated numerical tool has been employed to limit the effect of this phenomenon in order to enable the use of time-averaging techniques for noise mea- 
surement. Then, the noise due to camera sensor is heteroscedastic. It means that its variance is not a constant metrological characteristic since it depends on the grey level. Characterizing this noise and applying the so-called generalised Anscombe transform enabled us to obtain a homoscedastic noise in the grid images and to check that the predicting equations were actually satisfied.

Further work should be undertaken to observe if it is possible, for a given camera and for given data acquiring conditions, to characterise reliably once and for all the coefficients driving the GAT and to tabulate them. Another improvement should be to see if it is possible to characterise the coefficients of the GAT from one single grid image instead of a series of images, as suggested in Ref [14]. Both procedures would enable the user to predict the metrological performance in terms of resolution and spatial resolution for displacement and strain without the need to perform repeated acquisitions of grid images for each new test. 


\section{References}

[1] Sensicam QE-1288 datasheet, technical report, 2005. Technical report, PCO Imaging.

[2] P. Abrahamsen. A review of Gaussian random fields and correlation functions. Technical report, Norwegian Computing Center, Oslo, 1997.

[3] F.J. Anscombe. The transformation of Poisson, binomial and negative-binomial data. Biometrika, 35(3-4):246-254, 1948.

[4] C. Badulescu, M. Grédiac, J. Mathias, and D. Roux. A procedure for accurate onedimensional strain measurement using the grid method. Experimental Mechanics, 49:841-854, 2009.

[5] C. Badulescu, M. Grédiac, and J.-D. Mathias. Investigation of the grid method for accurate in-plane strain measurement. Measurement Science and Technology, 20(9):095102, 2009.

[6] C. Badulescu, M. Grédiac, J.-D. Mathias, and D. Roux. A procedure for accurate one-dimensional strain measurement using the grid method. Experimental Mechanics, 49(6):841-854, 2009.

[7] J. Boulanger, C. Kervrann, and P. Bouthemy. An adaptive statistical method for 4d-fluorescence image sequence denoising with spatio-temporal discontinuities preserving. In F. Faugel, P. Smigielski, A. Brandenburg, and J. Fontaine, editors, Biophotonics for Life Sciences and Medicine, pages 97-113. Fontis Media SA, 2006.

[8] J. Boulanger, C. Kervrann, P. Bouthemy, P. Elbau, J.-B. Sibarita, and J. Salamero. Patch-based nonlocal functional for denoising fluorescence microscopy image sequences. IEEE Transaction on Medical Imaging, 29(2):442-454, 2010.

[9] C.L. Chiang. Statistical methods of analysis. World Scientific Pub Co Inc., 2003.

[10] A. Chrysochoos and Y. Surrel. Chapter 1. Basics of metrology and introduction to techniques. In M. Grédiac and F. Hild, editors, Full-field measurements and identification in solid mechanics, pages 1-29. Wiley, 2012.

[11] S. Delpretti, F. Luisier, S. Ramani, T. Blu, and M. Unser. Multiframe sure-let denoising of timelapse fluorescence microscopy images. In Proceedings of the IEEE International Symposium on Biomedical Imaging (ISBI), pages 149-152, 2008.

[12] H. Faraji and W.J. MacLean. CCD noise removal in digital images. IEEE Transactions on Image Processing, 15(9):2676-2685, 2006. 
[13] A. Foi, S. Alenius, V. Katvonik, and K. Egiazarian. Noise measurement for rawdata of digital imaging sensors by automatic segmentation of non-uniform targets. IEEE Sensors Journal, 7(10):1456-1461, 2007.

[14] A. Foi, M. Trimeche, V. Katkovnik, and K. Egiazarian. Practical PoissonianGaussian noise modeling and fitting for single-image raw-data. IEEE Transactions on Image Processing, 17(10):1737-1754, 2008.

[15] R.C. Gonzalez and R.E. Woods. Digital Image Processing (3rd Edition). PrenticeHall, 2006.

[16] M. Grédiac, F. Sur, C. Badulescu, and J.-D. Mathias. Using deconvolution to improve the metrological performance of the grid method. Optics and Lasers in Engineering, 51(6):716-734, 2013.

[17] M. Grédiac and E. Toussaint. Studying the mechanical behaviour of asphalt mixtures with the grid method. Strain. An International Journal for Experimental Mechanics, 49(1):1-15, 2013. Wiley.

[18] E. Hack and J. Burke. Measurement uncertainty of linear phase-stepping algorithms. Review of Scientific Instruments, 82:061101, 2011.

[19] H. Haddadi and S. Belhabib. Use of a rigid-body motion for the investigation and estimation of the measurement errors related to digital image correlation technique. Optics and Lasers in Engineering, 46:185-196, 2008.

[20] G.E. Healey and R. Kondepudy. Radiometric CCD camera calibration and noise estimation. IEEE Transactions on Pattern Analysis and Machine Intelligence, 16(3):267-276, 1994.

[21] F. Hild and S. Roux. Comparison of local and global approaches to digital image correlation. Experimental Mechanics, 52(9):1503-1519, 2012.

[22] X. D. Ke, H. W. Schreier, M. A. Sutton, and Y.-Q. Yang. On error assessment in stereo-based deformation. part 2: experimental validation of uncertainty and biais estimates. Experimental Mechanics, 51:423-441, 2011.

[23] M. Makitalo and A. Foi. Optimal inversion of the generalized Anscombe transformation for Poisson-Gaussian noise. IEEE Transactions on Image Processing, 22(1):91-103, 2013.

[24] S. Mallat. A Wavelet Tour of Signal Processing, Third Edition: The Sparse Way. Academic Press, third edition, 2008.

[25] J. Molimard and L. Navarro. Uncertainty on fringe projection technique: A MonteCarlo-based approach. Optics and Lasers in Engineering, 51(7):840-847, 2013. 
[26] F. Murthagh, J.L. Starck, and A. Bijaoui. Image restoration with noise suppression using a multiresolution support. Astronomy and astrophysics, 112:179-189, 1995.

[27] V. Petrauskiene, A. Aleksa, A. Fedaravicius, and M. Ragulskis. Dynamic visual cryptography for optical control of vibration generation equipment. Optics and Lasers in Engineering, 50(6):869-876, 2012.

[28] J.L. Piro and M. Grédiac. Producing and transferring low-spatial-frequency grids for measuring displacement fields with moiré and grid methods. Experimental Techniques, 28(4):23-26, 2004.

[29] M. Ragulskis, R. Maskeluinas, and L. Saunoriene. Identification of in-plane vibrations using time average stochastic moiré. Experimental Techniques, 29(6):41-45, 2005 .

[30] M. Ragulskis, M. A. F. Sanjuan, and L. Saunoriene. Applicability of time average moiré techniques for chaotic oscillations. Physical Review E, 76:036208, 2007.

[31] S. Ramani, C. Vonesch, and M. Unser. Deconvolution of 3d fluorescence micrographs with automatic risk minimization. In Proceedings of the IEEE International Symposium on Biomedical Imaging (ISBI), pages 732-735, 2008.

[32] P.L. Reu. A study of the influence of calibration uncertainty on the global uncertainty for digital image correlation using a Monte Carlo approach. Experimental Mechanics, 53(9):1661-1680, 2013.

[33] S. Roux and F. Hild. Stress intensity factor measurements from digital image correlation : post-processing and integrated approaches. International Journal of Fracture, 140(1-4):141-157, 2006.

[34] F. Sur and M. Grédiac. Enhancing with deconvolution the metrological performance of the grid method for in-plane strain measurement. In Proceedings of the IEEE International Conference on Acoustics, Speech, and Signal Processing (ICASSP), pages 1563-1567, Vancouver, British Columbia, Canada, 2013.

[35] F. Sur and M. Grédiac. Sensor noise modeling by stacking pseudo-periodic grid images affected by vibrations. IEEE Signal Processing Letters, 21(4):432-436, 2014.

[36] F. Sur and M. Grédiac. Towards deconvolution to enhance the grid method for in-plane strain measurement. AIMS Inverse Problems and Imaging, 8(1):259-291, 2014.

[37] Y. Surrel. Photomechanics, Topics in Applied Physic 77, chapter Fringe Analysis, pages 55-102. Springer, 2000. 
[38] Y. Q. Wang, M. A. Sutton, H. A. Bruck, and H. W. Schreier. Statistical analysis of the effect of intensity pattern noise on the displacement measurement precision of digital image correlation using self-correlated images. Experimental Mechanics, 41(2):701-707, 2007.

[39] Y. Q. Wang, M. A. Sutton, H. A. Bruck, and H. W. Schreier. Quantitative error assessment in pattern matching: effects of intensity pattern noise, interpolation, strain and image contrast on motion measurements. Strain, 45(2):160-178, 2009.

[40] Y.-Q. Wang, M. A. Sutton, X. D. Ke, H. W. Schreier, P.L. Reu, and T.J. Miller. On error assessment in stereo-based deformation. part 1: theoretical developments for quantitative estimates. Experimental Mechanics, 51:405-422, 2011. 


\section{Appendices}

\section{A Noise variance in phase and phase derivative maps}

The grid image $s$ is impaired by noise. The phase and phase derivative maps are consequently also impaired by noise. In [36], a thorough computation has led to characterise the noise on the phase and phase derivative maps, assuming that the grid image is impaired by an additive Gaussian white noise $n$ of variance $v_{\text {image }}$. For the sake of completeness, this section gives a heuristic demonstration of the results of [36].

Let us note $\widetilde{n}$ the noise process on the phase and $\partial \widetilde{n} / \partial$. the noise process on the phase derivatives inherited from the noise process $n$ on the grid image. From now on, we use the discretised WFT which actually implements Equation (4) (without loss of generality, we consider the $\theta=0$ case). Since it is linear, $\Psi$ transforms into $\Psi_{n}=\Psi+\widehat{n}$ in the presence of an additive noise, where:

$$
\widehat{n}(\xi, \eta)=\sum_{i, j} n\left(x_{i}, y_{j}\right) g_{\sigma}\left(x_{i}-\xi, y_{j}-\eta\right) e^{-2 i \pi f x_{i}} \Delta_{x} \Delta_{y}
$$

Here $\left(\Delta_{x}, \Delta_{y}\right)$ is the grid pitch (here $\Delta_{x}=\Delta_{y}=1$ pixel).

A straightforward yet long calculation [36] proves that, if $\sigma \geq 1 / f)$, then $\operatorname{Re}(\widehat{n})$ and $\operatorname{Im}(\widehat{n})$ are uncorrelated Gaussian variables and are both wide-sense stationary processes with a covariance given by:

$$
\begin{array}{r}
\operatorname{Covar}\left(\operatorname{Re}(\widehat{n}(\xi, \eta)), \operatorname{Re}\left(\widehat{n}\left(\xi^{\prime}, \eta^{\prime}\right)\right)\right)=\operatorname{Covar}\left(\operatorname{Im}(\widehat{n}(\xi, \eta)), \operatorname{Im}\left(\widehat{n}\left(\xi^{\prime}, \eta^{\prime}\right)\right)\right) \\
\quad=\frac{v_{\text {image }} \Delta_{x} \Delta_{y}}{8 \pi \sigma^{2}} e^{-\left(\xi-\xi^{\prime}\right)^{2} /\left(4 \sigma^{2}\right)-\left(\eta-\eta^{\prime}\right)^{2} /\left(4 \sigma^{2}\right)}
\end{array}
$$

\section{Noise on the phase}

Since $\arg \left(\Psi_{n}\right)=\arctan \left(\frac{\operatorname{Im}(\Psi)+\operatorname{Im}(\widetilde{n})}{\operatorname{Re}(\Psi)+\operatorname{Re}(\widetilde{n})}\right)$, a first order Taylor expansion gives:

$$
\arg \left(\Psi_{n}\right)(\xi, \eta)=\arg (\Psi)(\xi, \eta)-\frac{\operatorname{Im}(\Psi(\xi, \eta, 0))}{|\Psi(\xi, \eta, 0)|^{2}} \operatorname{Re}(\widehat{n})(\xi, \eta)+\frac{\operatorname{Re}(\Psi(\xi, \eta, 0))}{|\Psi(\xi, \eta, 0)|^{2}} \operatorname{Im}(\widehat{n})(\xi, \eta)
$$

This first-order approximation turns out to be accurate enough for actual data.

Hence, $\widetilde{n}$ is approximately equal to $-\frac{\operatorname{Im}(\Psi(\xi, \eta, 0))}{|\Psi(\xi, \eta, 0)|^{2}} \operatorname{Re}(\widehat{n})(\xi, \eta)+\frac{\operatorname{Re}(\Psi(\xi, \eta, 0))}{|\Psi(\xi, \eta, 0)|^{2}} \operatorname{Im}(\widehat{n})(\xi, \eta)$. It is thus a 0 -mean spatially-correlated Gaussian random variable, and

$$
\begin{aligned}
\operatorname{Covar}(\widetilde{n}(\xi, \eta), & \left.\tilde{n}\left(\xi^{\prime}, \eta^{\prime}\right)\right)=\frac{\operatorname{Im}(\Psi(\xi, \eta, 0))}{|\Psi(\xi, \eta, 0)|^{2}} \frac{\operatorname{Im}\left(\Psi\left(\xi^{\prime}, \eta^{\prime}, 0\right)\right)}{\left|\Psi\left(\xi^{\prime}, \eta^{\prime}, 0\right)\right|^{2}} \operatorname{Covar}\left(\operatorname{Re}(\widehat{n}(\xi, \eta)), \operatorname{Re}\left(\widehat{n}\left(\xi^{\prime}, \eta^{\prime}\right)\right)\right. \\
& +\frac{\operatorname{Re}(\Psi(\xi, \eta, 0))}{|\Psi(\xi, \eta, 0)|^{2}} \frac{\operatorname{Re}\left(\Psi\left(\xi^{\prime}, \eta^{\prime}, 0\right)\right)}{\left|\Psi\left(\xi^{\prime}, \eta^{\prime}, 0\right)\right|^{2}} \operatorname{Covar}\left(\operatorname{Im}(\widehat{n}(\xi, \eta)), \operatorname{Im}\left(\widehat{n}\left(\xi^{\prime}, \eta^{\prime}\right)\right)\right)
\end{aligned}
$$


since $\operatorname{Re}(\widehat{n})$ and $\operatorname{Im}(\widehat{n})$ are uncorrelated.

With Equation 22, it is possible to conclude that:

$$
\begin{aligned}
\operatorname{Covar}\left(\widetilde{n}(\xi, \eta), \widetilde{n}\left(\xi^{\prime}, \eta^{\prime}\right)\right)= & \frac{v_{\text {image }} \cos \left(\arg (\Psi(\xi, \eta, 0))-\arg \left(\Psi\left(\xi^{\prime}, \eta^{\prime}, 0\right)\right)\right) \Delta_{x} \Delta_{y}}{8 \pi \sigma^{2}|\Psi(\xi, \eta)|\left|\Psi\left(\xi^{\prime}, \eta^{\prime}\right)\right|} \\
& \cdot e^{-\left(\xi-\xi^{\prime}\right)^{2} /\left(4 \sigma^{2}\right)-\left(\eta-\eta^{\prime}\right)^{2} /\left(4 \sigma^{2}\right)}
\end{aligned}
$$

Now it is shown in [36] and recalled in Section 3 that $\left|\Psi_{1}(\xi, \eta)\right| \simeq\left|d_{1}\right| \frac{\gamma A}{2}$ is a constant, denoted here by $K$. Since the phase variation are locally limited, $\arg (\Psi(\xi, \eta, 0)) \simeq$ $\arg \left(\Psi\left(\xi^{\prime}, \eta^{\prime}, 0\right)\right)$ and the cosine is approximately equal to 1 .

Consequently, the noise $\widetilde{n}$ on the phase map can be considered as a stationary spatially correlated process, with a variance given by the value of the covariance for $\xi=\xi^{\prime}$ and $\eta=\eta^{\prime}$ in Equation 25:

$$
\operatorname{Var}(\widetilde{n}(\xi, \eta))=\frac{v_{\text {image }} \Delta_{x} \Delta_{y}}{8 \pi \sigma^{2} K^{2}}
$$

\section{Noise on the phase derivatives}

With the same assumption as above, the noise process $\partial \widetilde{n} / \partial \cdot$ on the phase derivative is a stationary spatially correlated process, whose autocovariance function is the opposite of the second derivative of the autocovariance of $\widetilde{n}[2]$ :

$\operatorname{Covar}\left(\frac{\partial \widetilde{n}}{\partial \xi}(\xi, \eta), \frac{\partial \widetilde{n}}{\partial \xi}\left(\xi^{\prime}, \eta^{\prime}\right)\right)=\frac{v_{\text {image }} \Delta_{x} \Delta_{y}}{16 \pi \sigma^{4} K^{2}} \cdot e^{-\left(\xi-\xi^{\prime}\right)^{2} /\left(4 \sigma^{2}\right)-\left(\eta-\eta^{\prime}\right)^{2} /\left(4 \sigma^{2}\right)}\left(1-\frac{\left(\xi-\xi^{\prime}\right)^{2}}{2 \sigma^{2}}\right)$

and:

$\operatorname{Covar}\left(\frac{\partial \widetilde{n}}{\partial \eta}(\xi, \eta), \frac{\partial \widetilde{n}}{\partial \eta}\left(\xi^{\prime}, \eta^{\prime}\right)\right)=\frac{v_{\text {image }} \Delta_{x} \Delta_{y}}{16 \pi \sigma^{4} K^{2}} \cdot e^{-\left(\xi-\xi^{\prime}\right)^{2} /\left(4 \sigma^{2}\right)-\left(\eta-\eta^{\prime}\right)^{2} /\left(4 \sigma^{2}\right)}\left(1-\frac{\left(\eta-\eta^{\prime}\right)^{2}}{2 \sigma^{2}}\right)$

Consequently, the variance of the noise on phase derivative maps is obtained by imposing $\xi=\xi^{\prime}$ and $\eta=\eta^{\prime}$ in Equation 28:

$$
\operatorname{Var}\left(\frac{\partial \widetilde{n}}{\partial \xi}(\xi, \eta)\right)=\operatorname{Var}\left(\frac{\partial \widetilde{n}}{\partial \eta}(\xi, \eta)\right)=\frac{v_{\text {image }} \Delta_{x} \Delta_{y}}{16 \pi \sigma^{4} K^{2}}
$$

\section{B Influence of the line profile on the value of $\left|d_{1}\right|$}

The variance of the noise in the phase and phase derivative maps is inversely proportional to $K$ recalled in Equation 7:

$$
K=\frac{\left|d_{1}\right| \gamma A}{2}
$$


In particular, $K$ depends via $d_{1}$ on the line profile frng (which is a real $2 \pi$-periodic function with a peak-to-peak amplitude equal to 1 and average value 0 ). The larger $\left|d_{1}\right|$, the smaller the noise variance. We first show in this appendix that the largest value of $\left|d_{1}\right|$ is attained for a sine profile.

Let $d_{k}=1 /(2 \pi) \int_{-\pi}^{\pi} \operatorname{frng}(x) e^{-i n x} \mathrm{~d} x(k \in \mathbb{Z})$ be the Fourier coefficients of $\operatorname{frng}(x)$, and let $A^{2}=1 /(2 \pi) \int_{-\pi}^{\pi} \operatorname{frng}^{2}(x) \mathrm{d} x$ be the squared $L^{2}$ norm of frng $(x)$. Parseval's theorem states that $\sum_{k}\left|d_{k}\right|^{2}=A^{2}$. Here $d_{0}=0$ since the average of frng is 0 , and $d_{k}=$ $\overline{d_{-k}}$ since frng is a real function ( $\bar{z}$ denoting the conjugate of any complex number $z$ ). For a fixed $A,\left|d_{1}\right|$ attains thus its highest possible value if $d_{k}=0$ for any $k>1$. In this case $\left|d_{1}\right|^{2}=\left|d_{-1}\right|^{2}=A^{2} / 2$, and there exists $\phi \in \mathbb{R}$ such that $d_{1}=A e^{i \phi} / \sqrt{2}$ and $d_{-1}=A e^{-i \phi} / \sqrt{2}$.

As a consequence, $\operatorname{frng}(x)=d_{1} e^{i x}+d_{-1} e^{-i x}=2 A / \sqrt{2} \cos (x+\phi)$.

Since the extremum of frng is $1, A=\sqrt{2} / 2=1 / \sqrt{2}$.

We have thus demonstrated that a sine function gives the largest $\left|d_{1}\right|$, and that in this case: $\left|d_{1}\right|=1 / 2$.

However a real grid image is manufactured in such a way that the optimum sine profile is hard to achieve. A more realistic profile function frng is a rectangular function of width $a(0<a<2 \pi)$ such that for every $x \in[-\pi, \pi]$,

$$
\operatorname{frng}(x)=\left\{\begin{array}{l}
1-a /(2 \pi) \quad \text { if }|x|<a / 2 \\
-a /(2 \pi) \quad \text { otherwise }
\end{array}\right.
$$

so that its average value is 0 and its peak-to-peak amplitude is 1 .

The Fourier coefficient $d_{1}$ of this rectangular function is:

$$
\begin{aligned}
d_{1} & =\frac{1}{2 \pi} \int_{-\pi}^{\pi} \operatorname{frng}(x) e^{-i x} \mathrm{~d} x \\
& =\frac{1}{2 \pi} \int_{-a / 2}^{a / 2}\left(1-\frac{a}{2 \pi}\right) e^{-i x} \mathrm{~d} x-\frac{1}{2 \pi} \int_{-\pi}^{-a / 2} \frac{a}{2 \pi} e^{-i x} \mathrm{~d} x-\frac{1}{2 \pi} \int_{a / 2}^{\pi} \frac{a}{2 \pi} e^{-i x} \mathrm{~d} x \\
& =\frac{1}{\pi} \sin \left(\frac{a}{2}\right)
\end{aligned}
$$

Consequently, the rectangular profile giving the largest $\left|d_{1}\right|$ is such that $a=\pi$ (i.e., the black ink covers half of the pattern pitch). In this case, $\left|d_{1}\right|=1 / \pi \simeq 0.32$ which is $36 \%$ smaller than in the sine profile case where $\left|d_{1}\right|=0.5$. For information, the $\sin ^{3}(x)$ profile which is used to generate the synthetic grid images in [36][16] is such that $\left|d_{1}\right|=3 / 8=0.375$. 


\section{Effect of bilinear interpolation on noise variance in phase and phase derivative maps}

Let $u$ be either the phase map or the phase derivative map. The process described in [5] to map the phase map before and after deformation in the same coordinate system uses a bilinear interpolation [15] to get the value of $u$ at non-integer coordinates. These interpolated values are a linear combination of the values of $u$ at nearby integer coordinates. Hence the noise term is also a linear combination $\sum_{i} a_{i} n_{i}$ where $i$ spans the four adjacent pixels and $0 \leq a_{i} \leq 1$ are such that $\sum_{i} a_{i}=1$.

The variance of the noise in the interpolated map then writes:

$$
\sum_{i} a_{i}^{2} \operatorname{Var}\left(n_{i}\right)+\sum_{i \neq j} a_{i} a_{j} \operatorname{Covar}\left(n_{i}, n_{j}\right)
$$

It is shown in [36] and recalled in appendix A that the covariance of the noise on the phase map is:

$$
\operatorname{Covar}\left(n_{i}, n_{j}\right)=\frac{v_{\text {image }} \Delta_{x} \Delta_{y}}{8 \pi \sigma^{2} K^{2}} e^{-\|i-j\|^{2} /\left(4 \sigma^{2}\right)}
$$

and on the phase derivative map ( $\xi$-derivative):

$$
\operatorname{Covar}\left(n_{i}, n_{j}\right)=\frac{v_{\text {image }} \Delta_{x} \Delta_{y}}{16 \pi \sigma^{4} K^{2}} e^{-\|i-j\|^{2} /\left(4 \sigma^{2}\right)}\left(1-\frac{\left(\xi-\xi^{\prime}\right)^{2}}{2 \sigma^{2}}\right)
$$

where $\|i-j\|$ denotes the Euclidean distance between coordinates $i$ and $j$.

Since $\|i-j\| \in\{0,1, \sqrt{2}\}$ pixel and since the value of $\sigma$ is typically at least equal to 5 pixels, $e^{-\|i-j\|^{2} /\left(4 \sigma^{2}\right)} \simeq 1$ and $\left(\xi-\xi^{\prime}\right)^{2} /\left(2 \sigma^{2}\right) \simeq 0$. Concerning the phase noise, the covariance simplifies into:

$$
\operatorname{Covar}\left(n_{i}, n_{j}\right) \simeq \frac{v_{\text {image }} \Delta_{x} \Delta_{y}}{8 \pi \sigma^{2} K^{2}}=\operatorname{Var}\left(n_{i}\right)
$$

Concerning the phase derivative noise:

$$
\operatorname{Covar}\left(n_{i}, n_{j}\right) \simeq \frac{v_{\text {image }} \Delta_{x} \Delta_{y}}{16 \pi \sigma^{4} K^{2}}=\operatorname{Var}\left(n_{i}\right)
$$

Hence, it is possible to conclude from Equations 35 and 38-39 that the variance of the noise after a bilinear interpolation, either in the phase map or in the phase derivative map, is

$$
\left(\sum_{i} a_{i}\right)^{2} \operatorname{Var}(n)=\operatorname{Var}(n)
$$

since by definition $\sum_{i} a_{i}=1$.

Consequently, the variance of the noise is the same in the raw phase and phase derivative maps as in the corresponding interpolated maps. The reason is that the range of the noise correlation is much larger than the size of the 4-pixel neighborhood used in bilinear interpolation. 


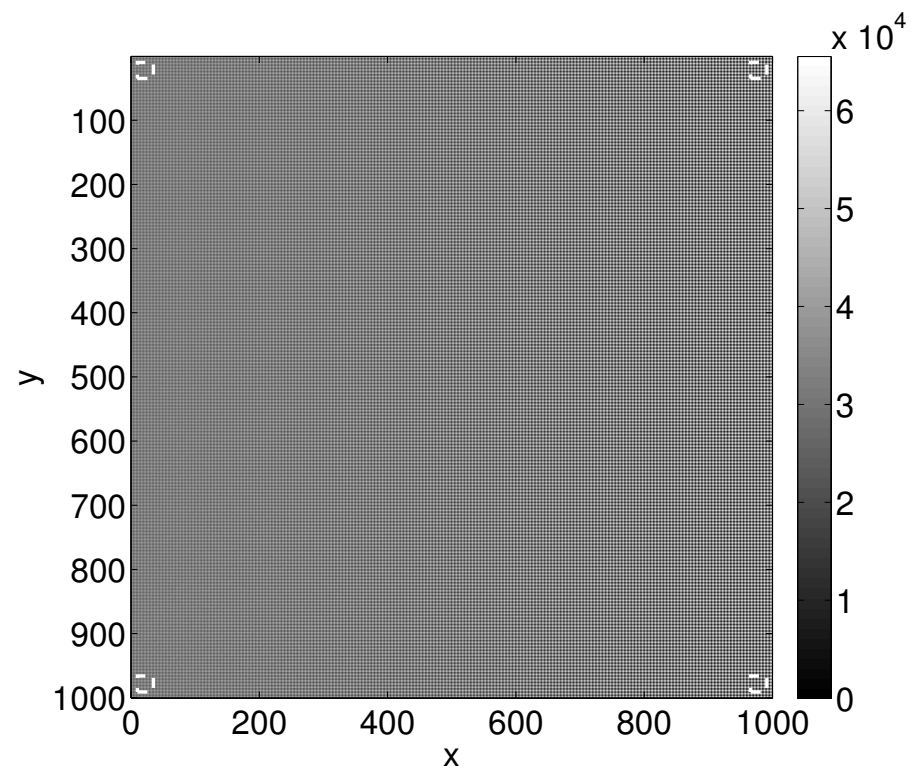

Figure 1: Simulated grid (the reader is invited to zoom this figure in the pdf file) 


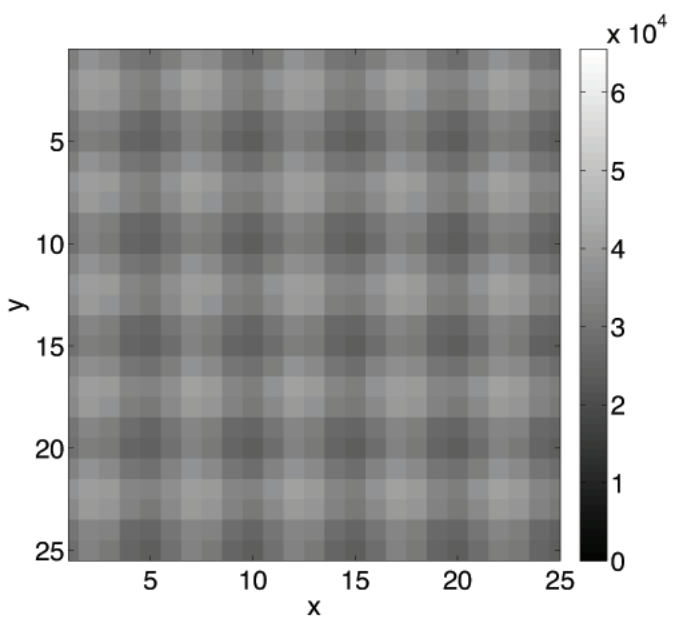

a- top left

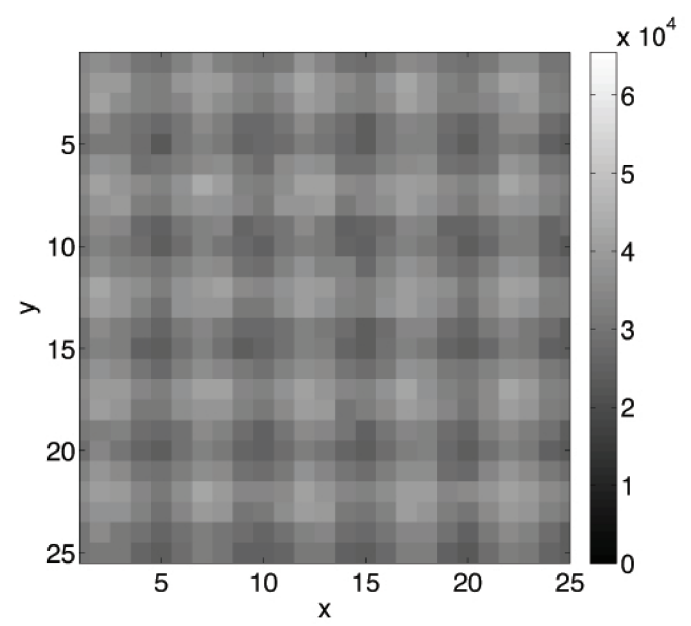

c- bottom left

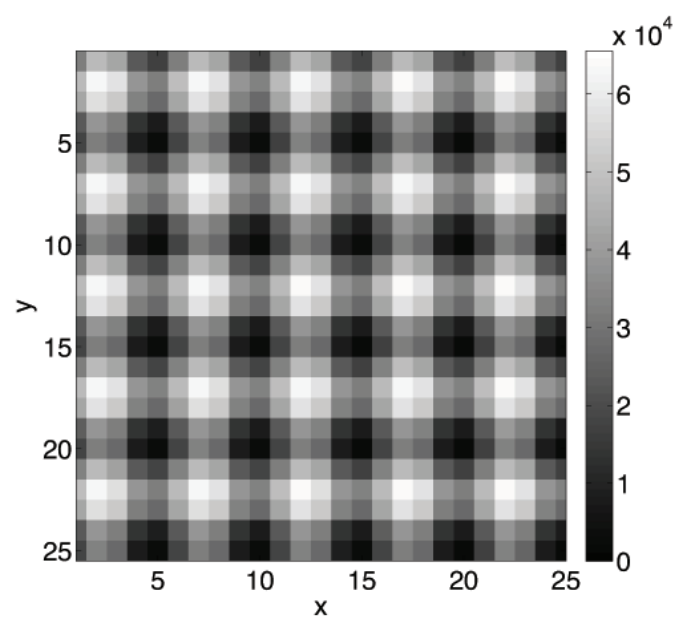

b-top right

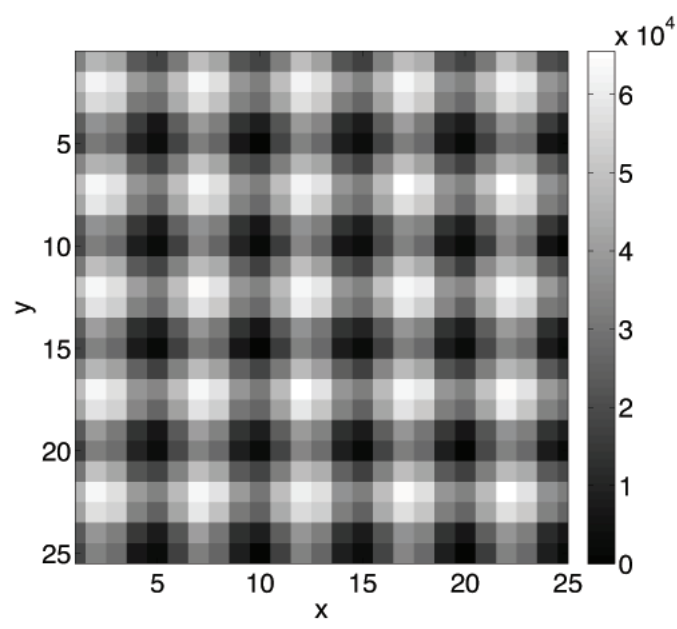

d-bottom right

Figure 2: Zoomed squares picked near the corners of the simulated grid 


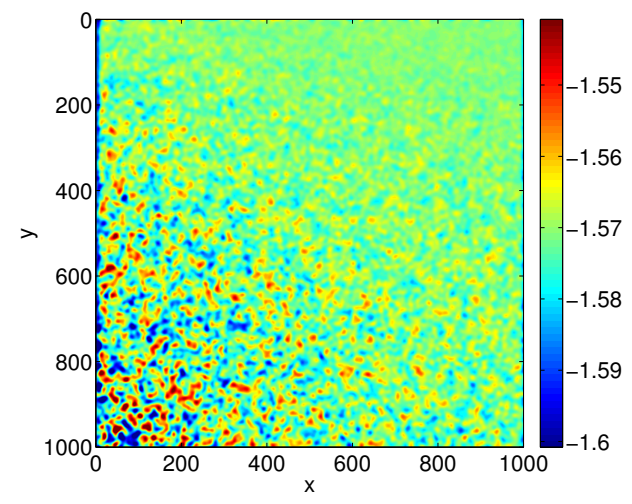

$\mathrm{a}-\sigma_{\phi}$

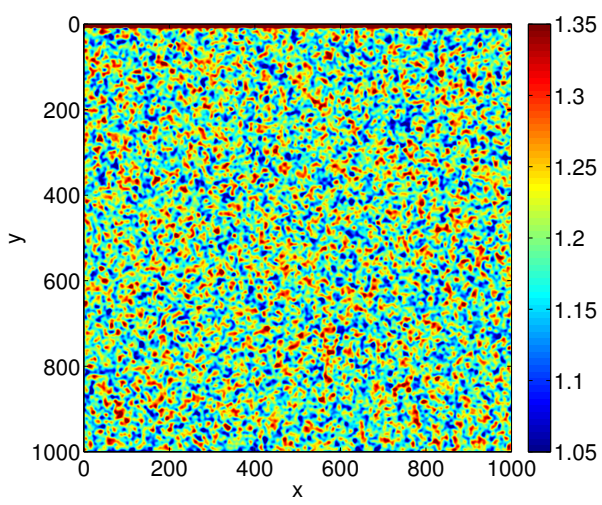

b- $r_{\phi}$. Predicted value: $\frac{3}{\sqrt{2 \pi}} \simeq 1.1968$ pixel

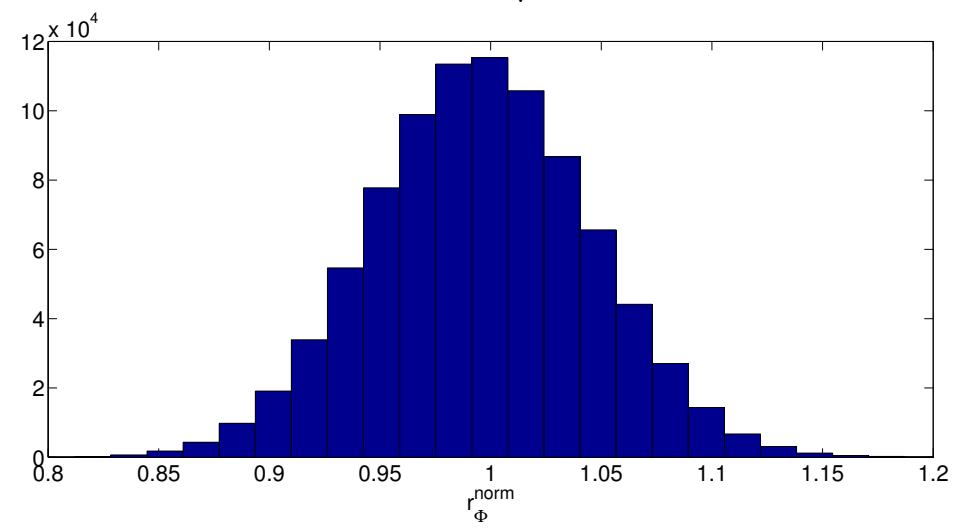

c- histogram of $r_{\phi}^{\text {norm }}=\frac{\sqrt{2 \pi}}{3} \times r_{\phi}$

Figure 3: Noise propagation from a simulated image to the phase maps. Noise features spatially changing variance and illumination in the grid images 


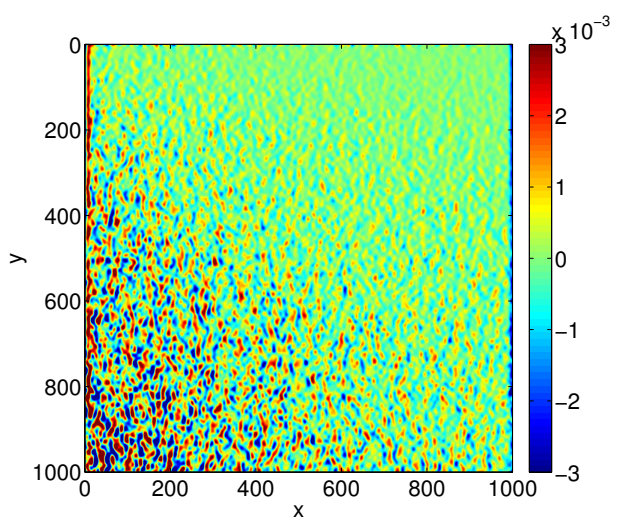

$\mathrm{a}-\sigma_{\phi^{\prime}}$

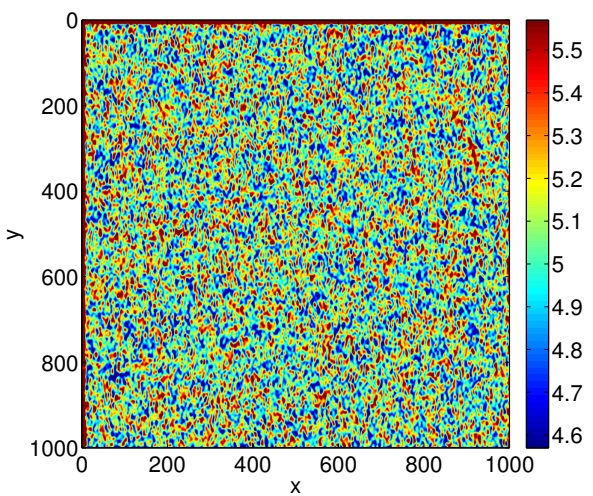

b- $r_{\phi^{\prime}}$. Predicted value: $\frac{9}{\sqrt{\pi}} \simeq 5.0777$ pixel

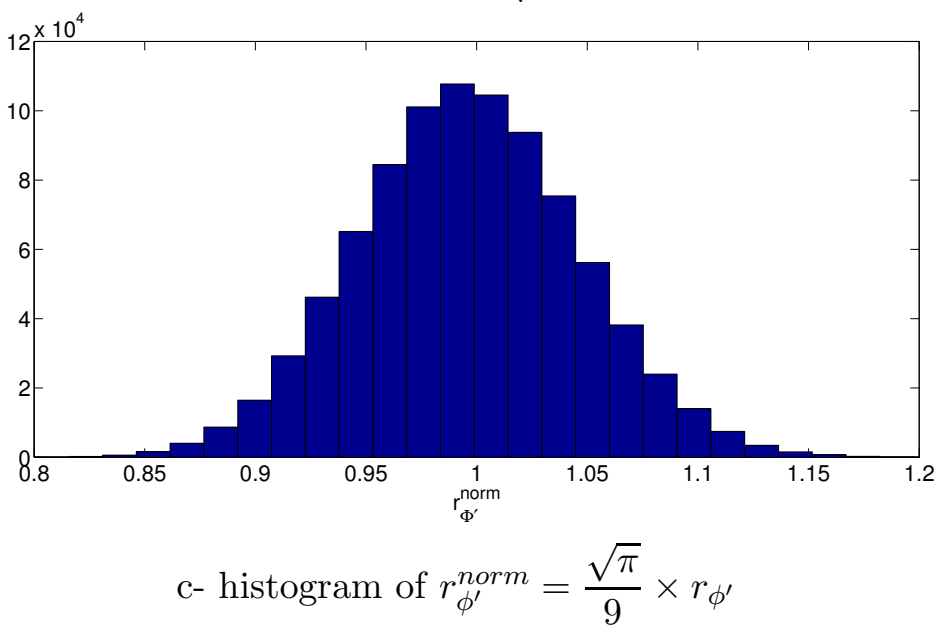

Figure 4: Noise propagation from a simulated image to the phase derivative maps. Noise features spatially changing variance and illumination in the grid images 


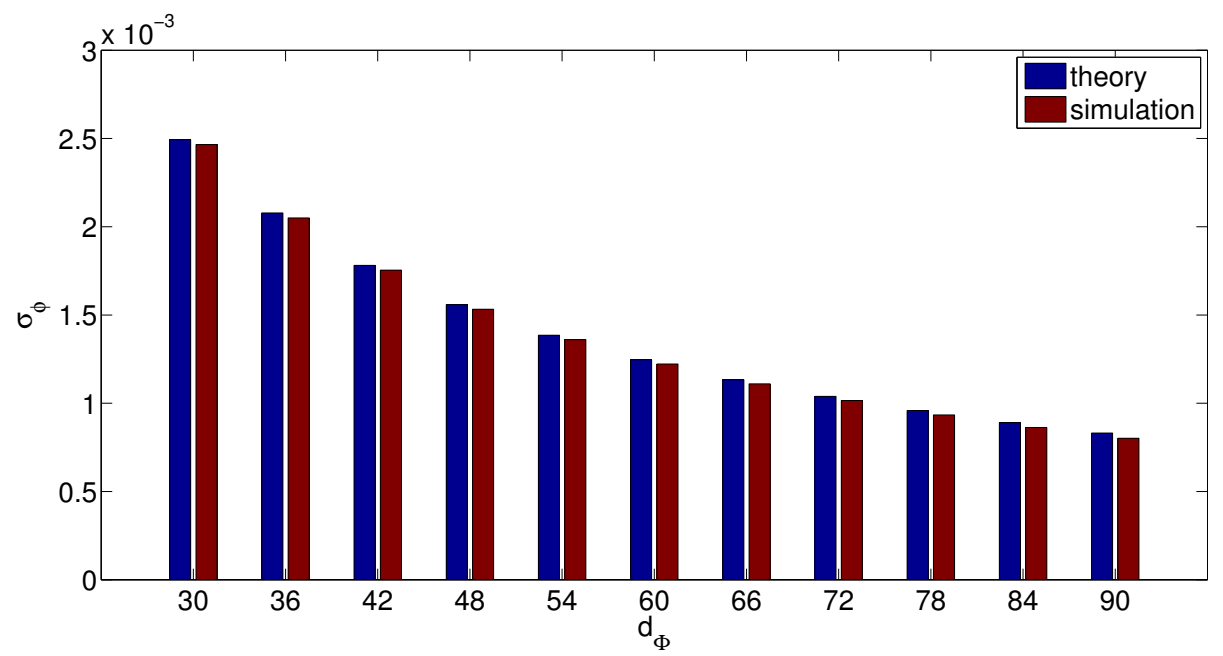

a- for the phase: $\sigma_{\phi_{x}}$ vs. $d_{\Phi}$

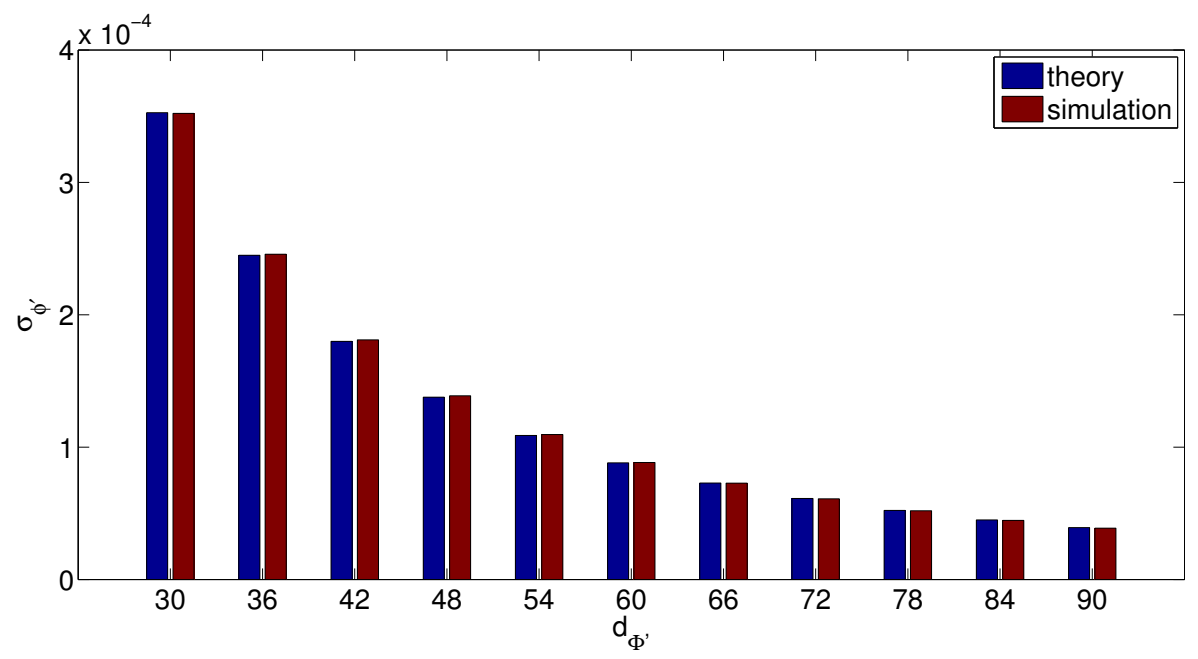

b- for the phase derivative: $\sigma_{\phi_{x}^{\prime}}$ vs. $d_{\Phi^{\prime}}$

Figure 5: Resolution vs. spatial resolution. Comparison between theory and simulation 


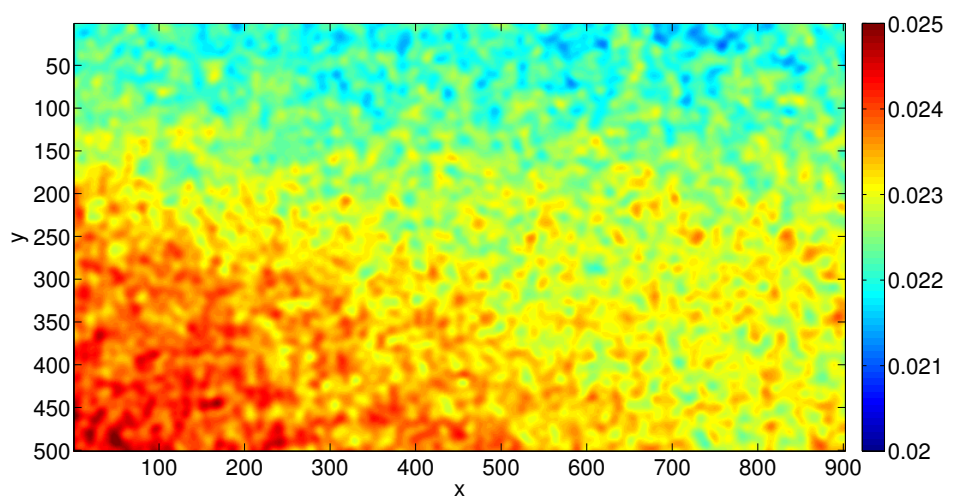

a- resolution identified with raw grid images

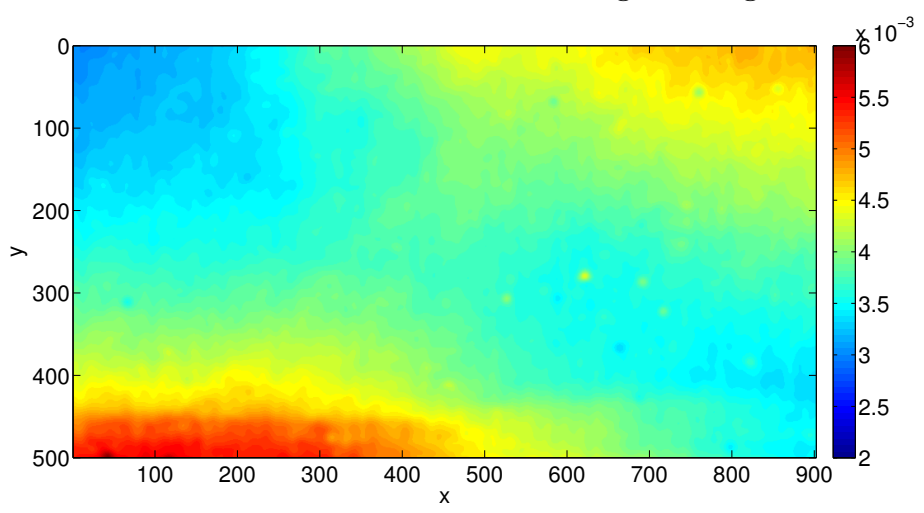

b- resolution predicted with Equation 10-a in which $\sigma_{\text {image }}=\sigma_{\text {image }}^{\text {equivalent }}=407$

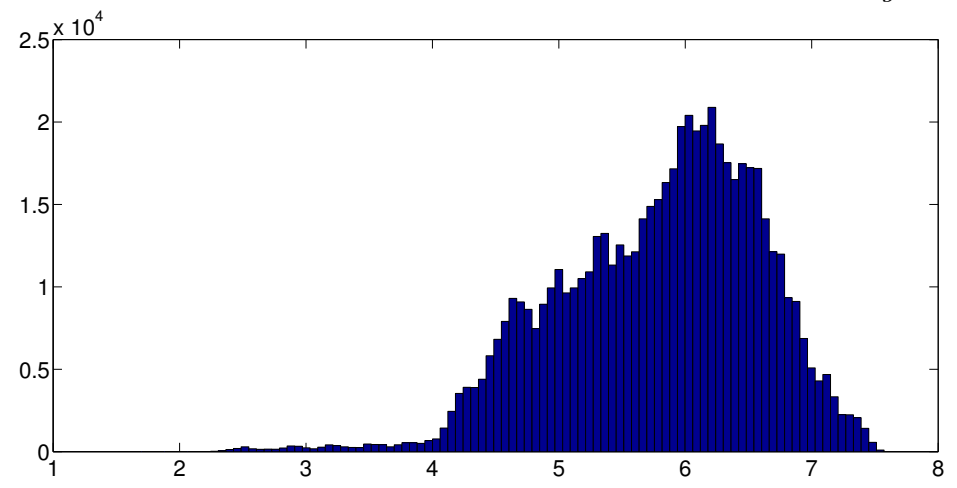

c- histogram of the ratio between a- and b-. Mean value $=5.92$

Figure 6: Displacement resolution in pixel (in micron by multiplying by 40). Imposed displacement $u=0.1 \mathrm{~mm}$. Identified and estimated distributions with $\sigma_{\text {image }}=$ $\sigma_{\text {image }}^{\text {equivalent }}=407$ 


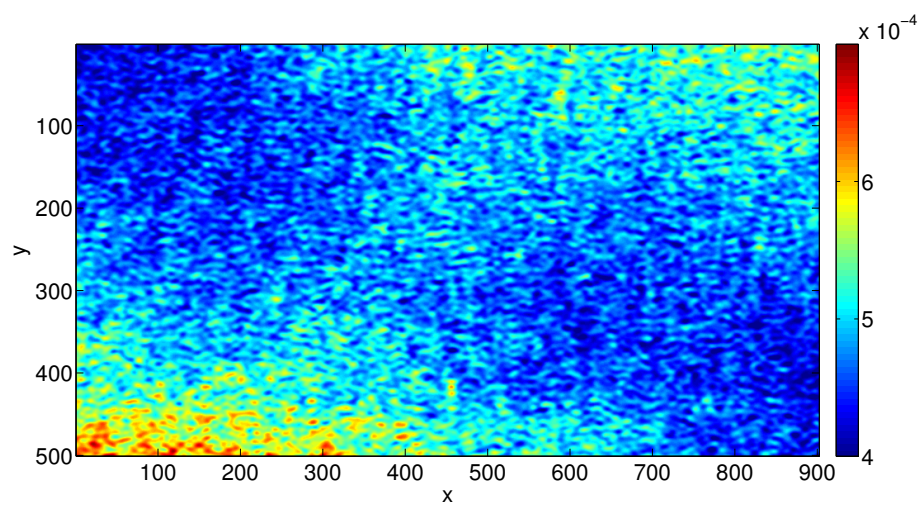

a- resolution identified with raw grid images

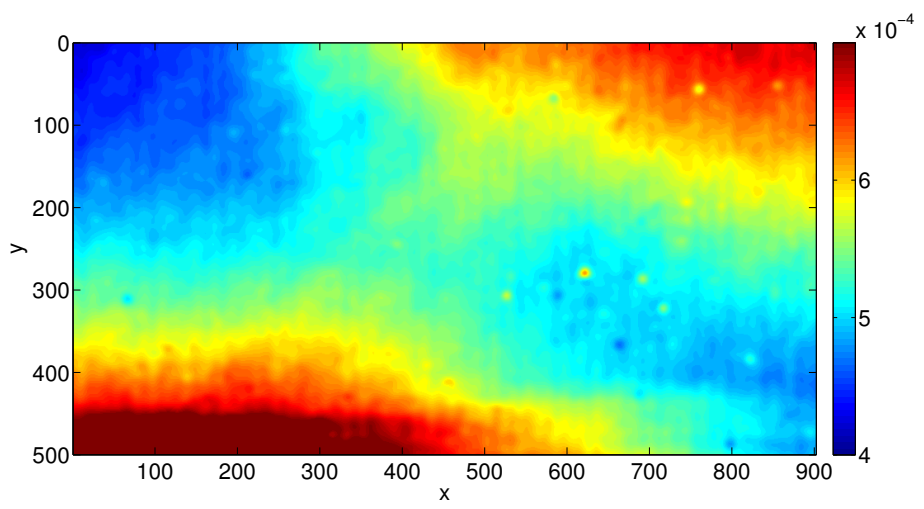

b- resolution predicted with Equation $10-\mathrm{b}$ in which $\sigma_{\text {image }}=\sigma_{\text {image }}^{\text {equivalent }}$

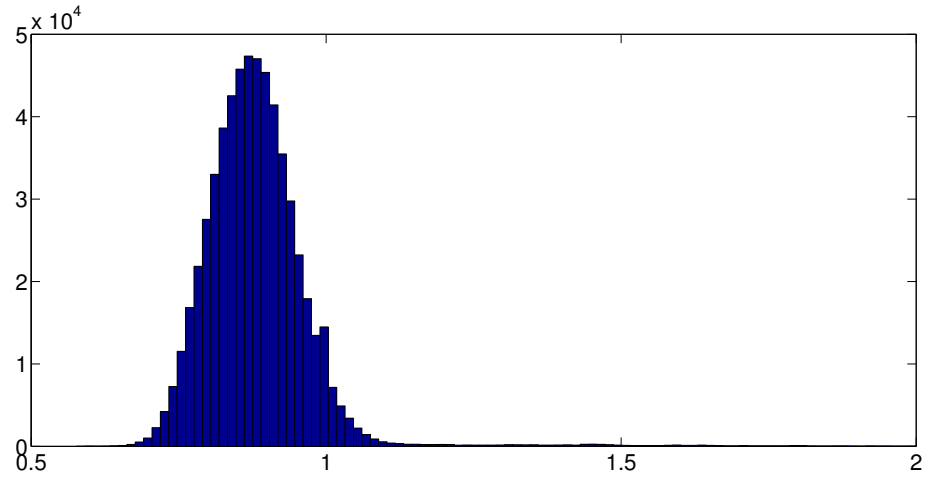

c- histogram of the ratio between a- and b-. Mean value $=0.87$

Figure 7: Strain resolution. Imposed displacement $u=0.1 \mathrm{~mm}$. Identified and estimated distributions with $\sigma_{\text {image }}=\sigma_{\text {image }}^{\text {equivalent }}$ 


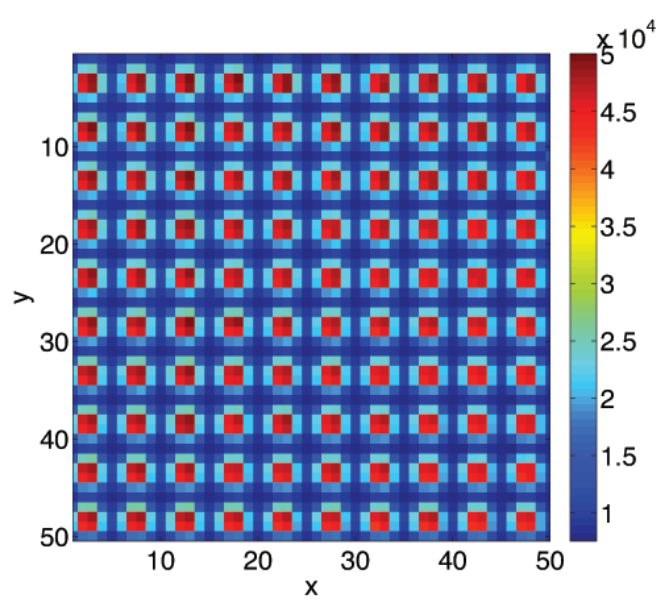

a- close-up view of the mean grid image

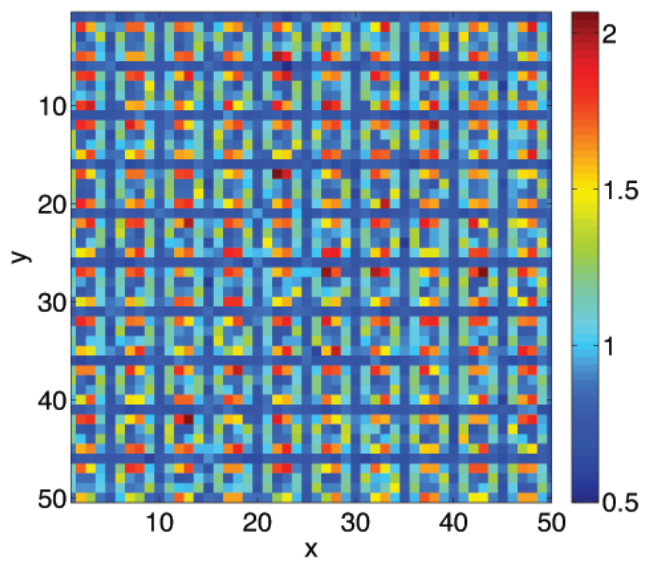

c- variance

after GAT but without NRSR

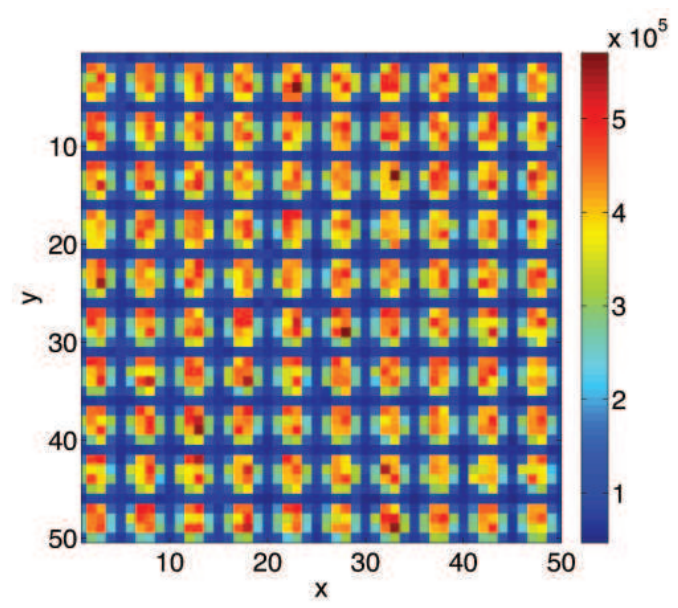

b- variance of the

200 images over the same zone

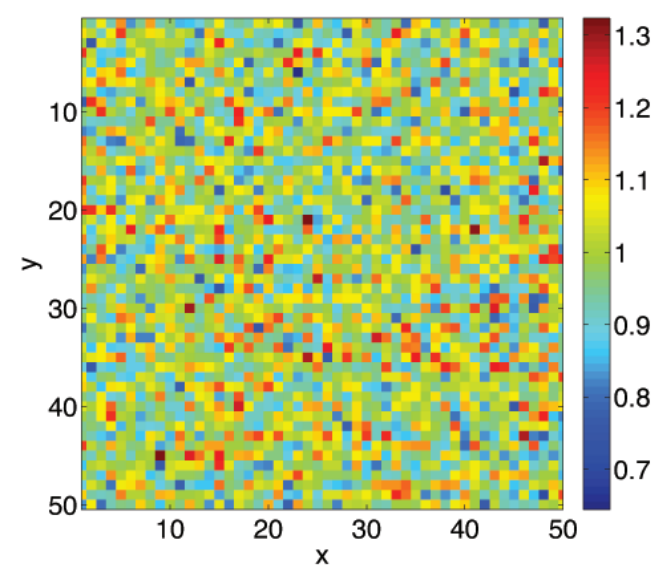

d- variance

after NRSR and GAT

Figure 8: Experimental evidence of homoscedascity. Benefit of NRSR and GAT 


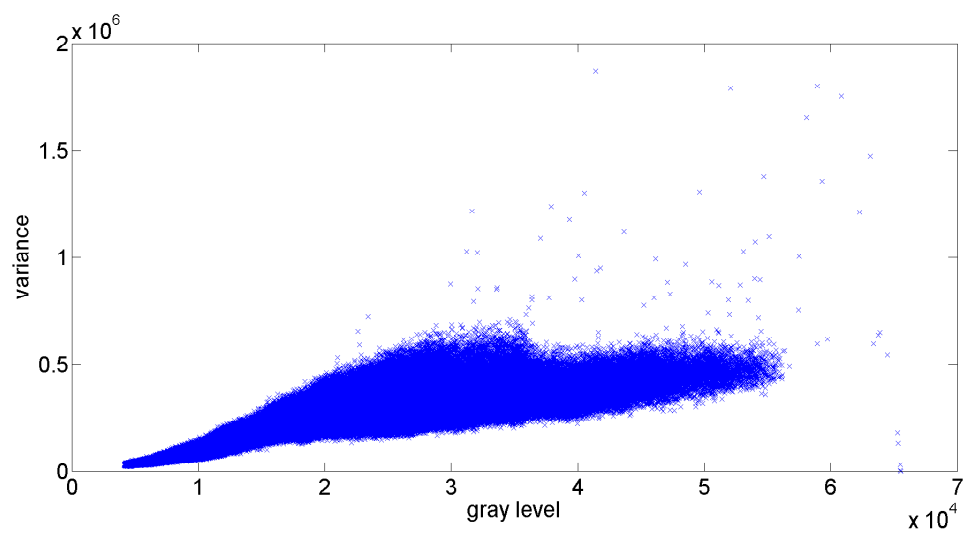

a- raw variance at all the pixels

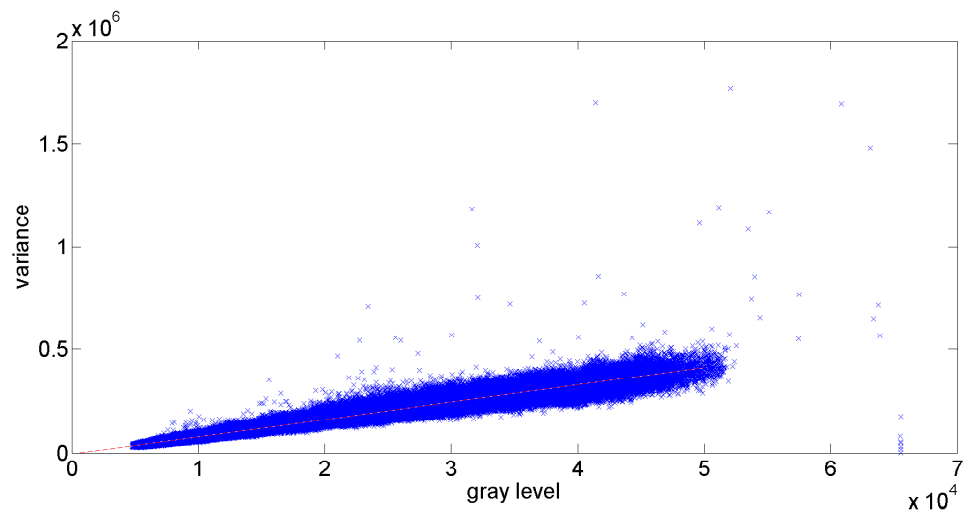

b- after reducing the non-random part of the signal

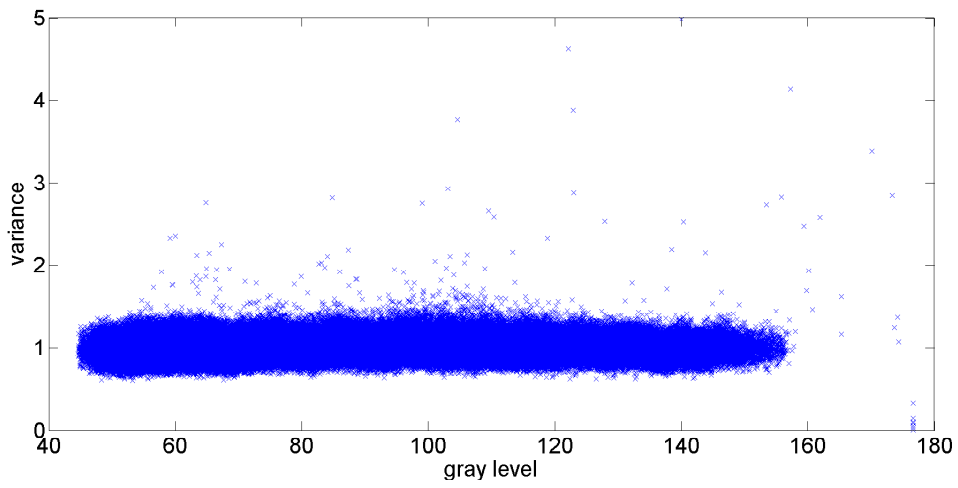

c- after reducing the non-random part of the signal and applying the Anscombe transform. The scales along the $x$ - and $y$-axes are different from above

Figure 9: Variance vs. grey level 


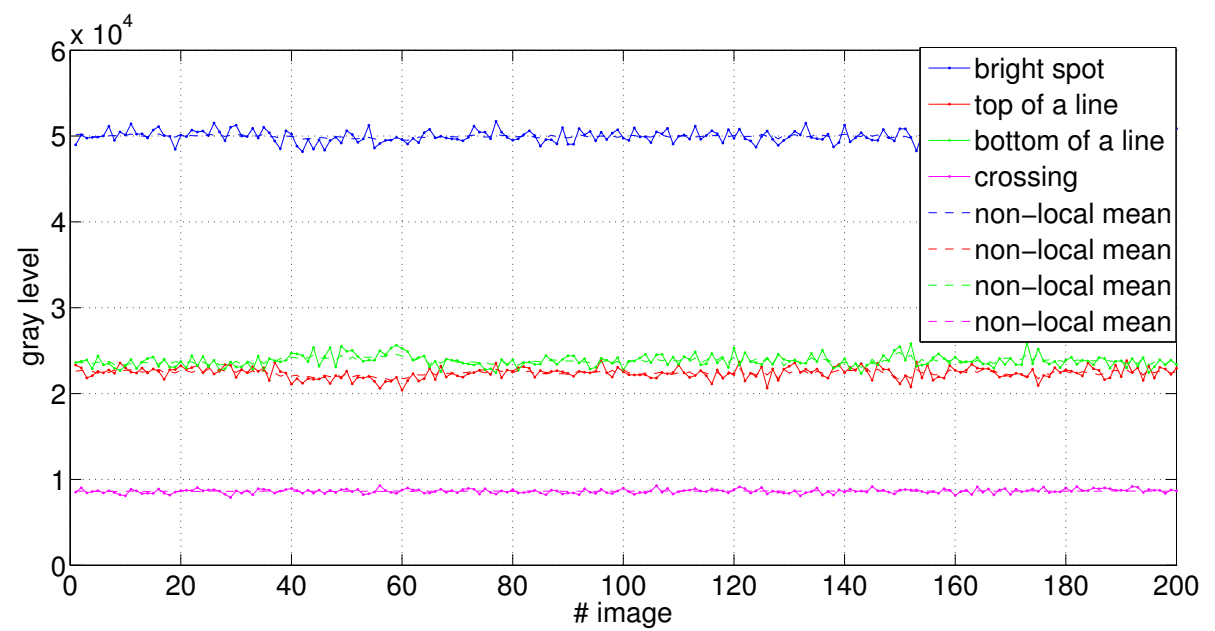

Figure 10: Temporal fluctuation of the grey level before NRSR at various types of pixels: bright spot, top of a horizontal line, bottom of a horizontal line, crossing 


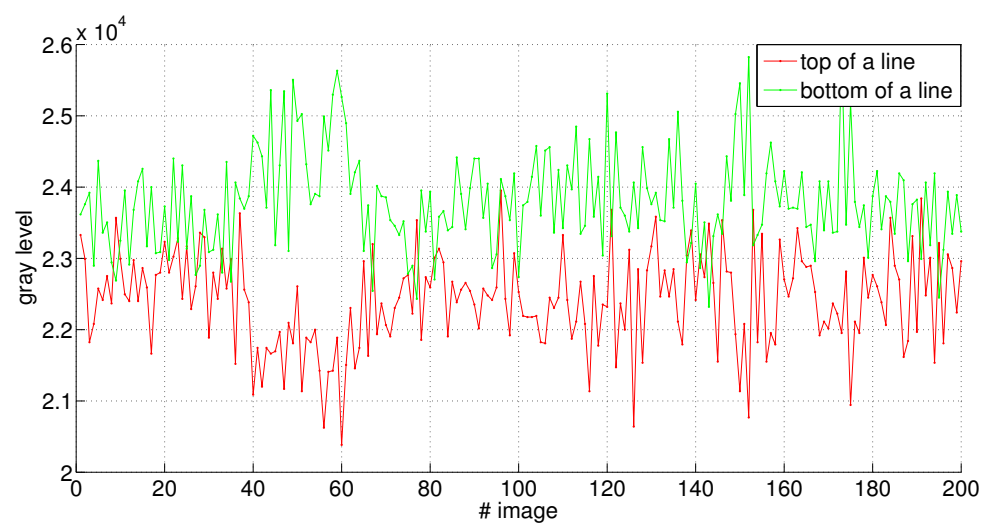

a- before NRSR

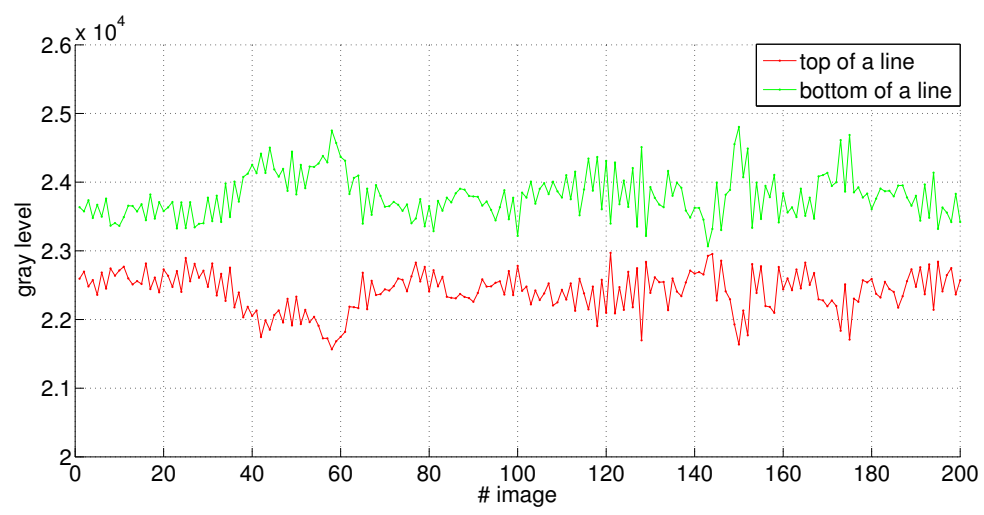

b- effect of micro-oscillations

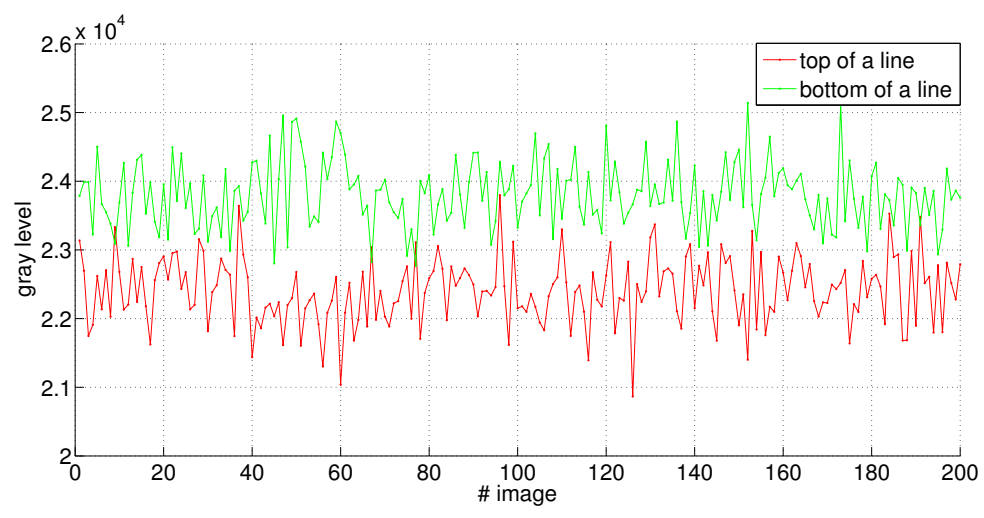

c- after NRSR

Figure 11: Effect of the NRSR on the grey level at the top and bottom of a horizontal line of the grid 


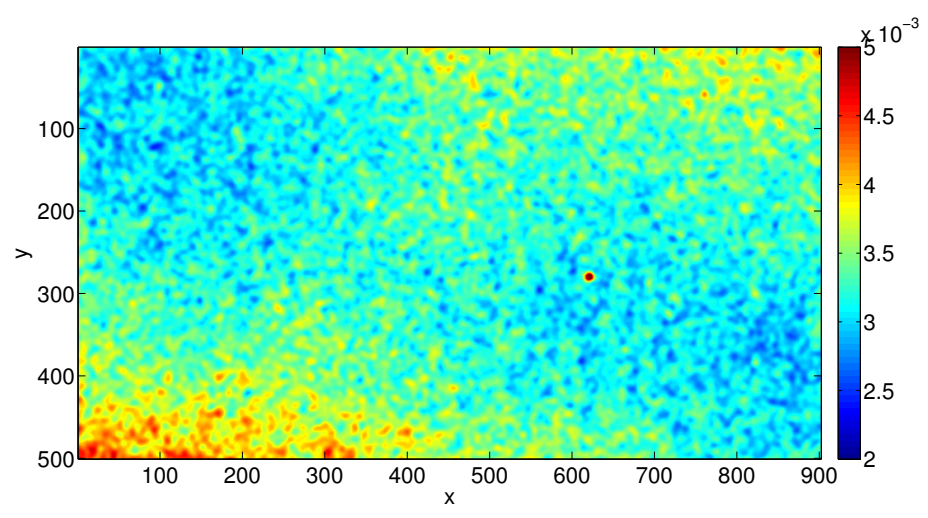

a- resolution identified with grid images after NRSR

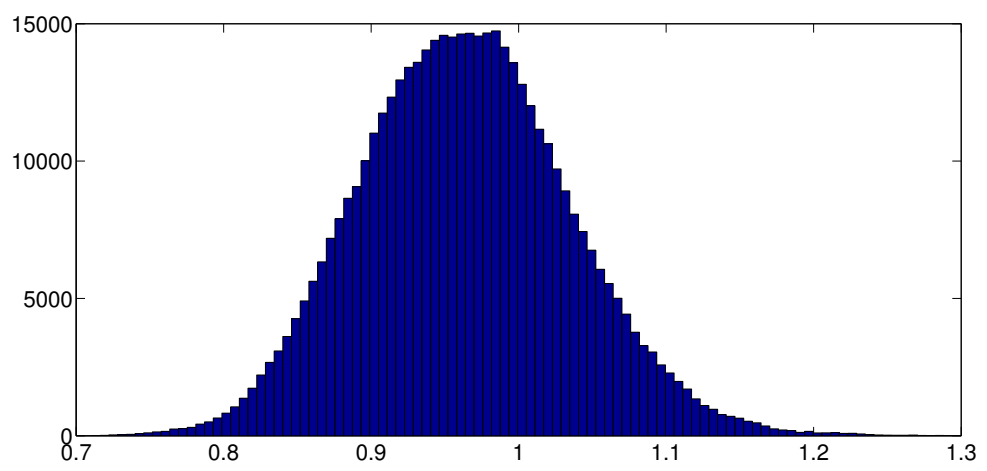

b- histogram of the ratio $\alpha$ between resolution identified with grid images after NRSR and resolution predicted with Equation 10-a in which $\sigma_{\text {image }}=\sigma_{\text {image }}^{\text {equivalent }}=352$. Mean value: 0.96 .

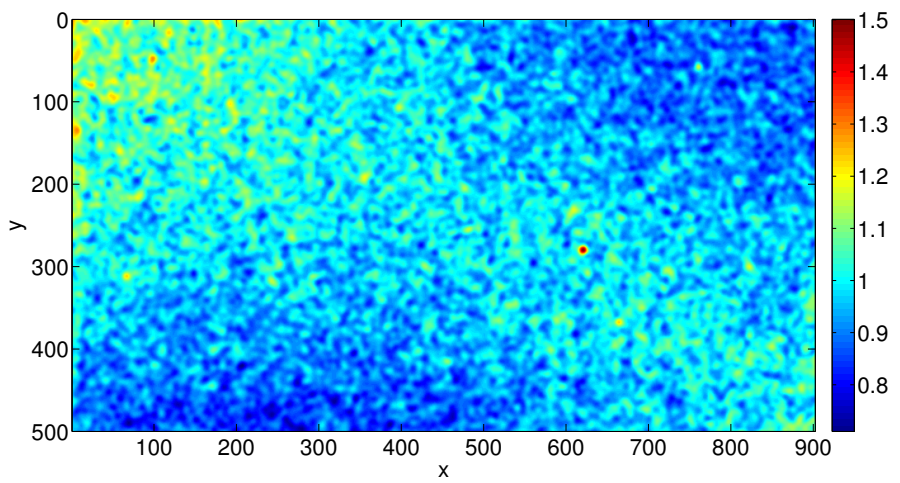

c- spatial distribution of $\alpha$

Figure 12: Displacement resolution after NRSR. Imposed displacement $u=0.1 \mathrm{~mm}$. Identified and estimated distributions with $\sigma_{\text {image }}=\sigma_{\text {image }}^{\text {equivalent }}=352$ 


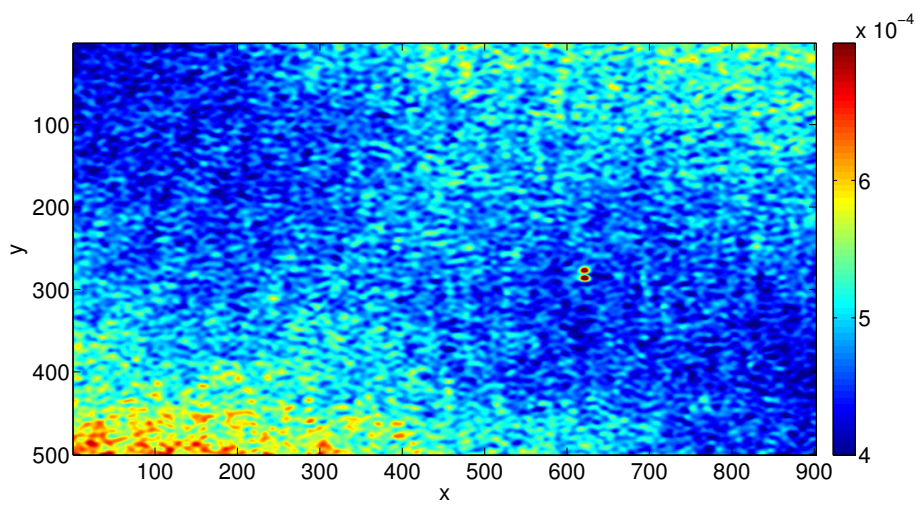

a- resolution identified with grid images after NRSR

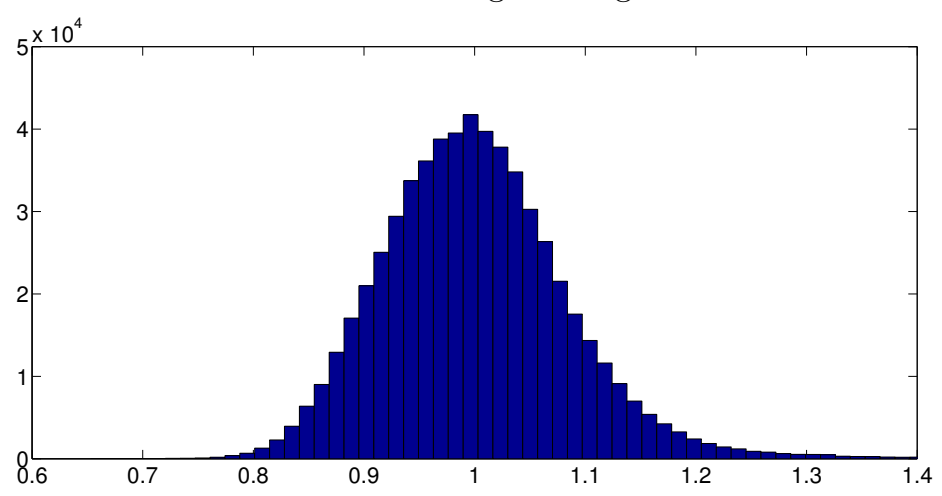

b- histogram of the ratio $\beta$ between resolution identified with grid images after NRSR and resolution predicted with Equation 10 -b in which $\sigma_{\text {image }}=\sigma_{\text {image }}^{\text {equivalent }}=352$. Mean value: 0.99 .

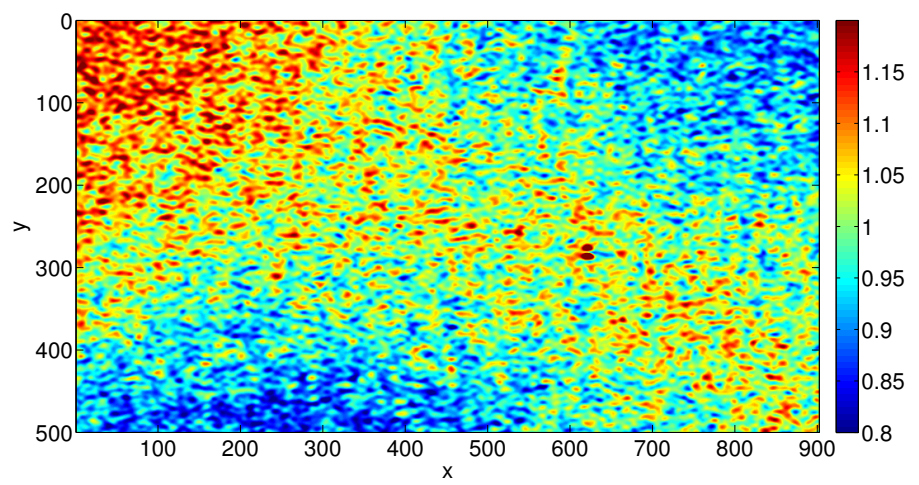

c- spatial distribution of $\beta$

Figure 13: Strain resolution after NRSR. Imposed displacement $u=0.1 \mathrm{~mm}$. Identified and estimated distributions with $\sigma_{\text {image }}=\sigma_{\text {image }}^{\text {equivalent }}=352$ 


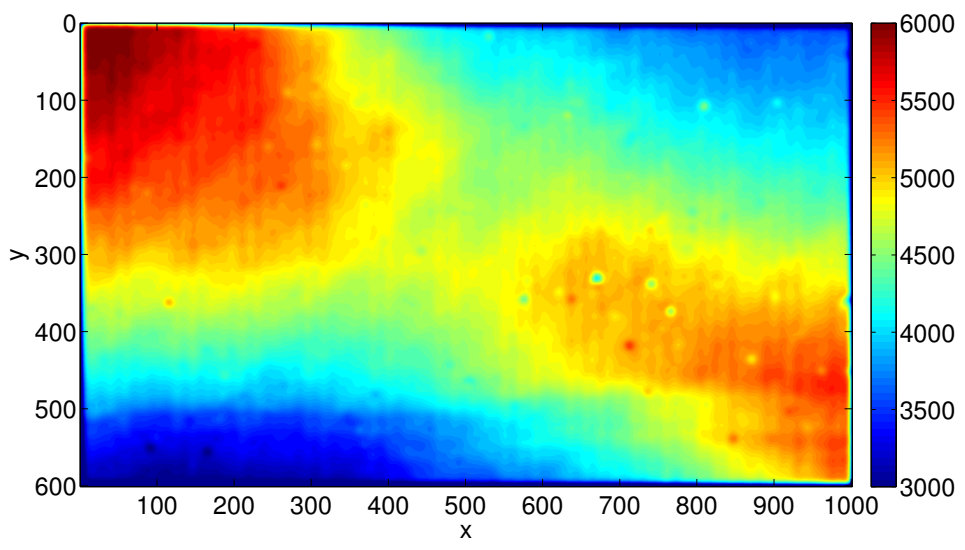

a- for the raw grid image

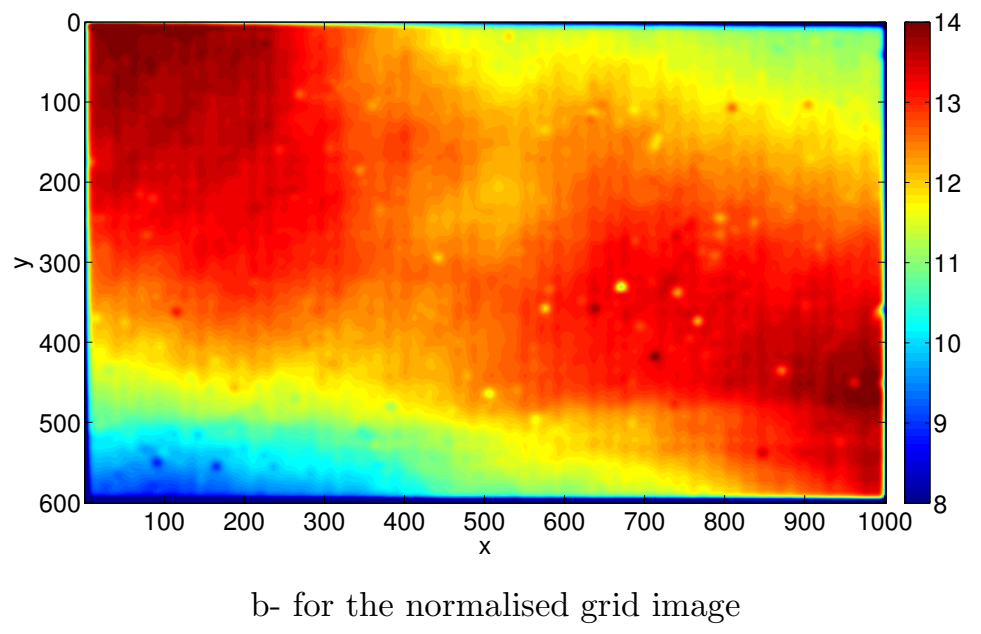

Figure 14: Mean value of the modulus $K$ of the WFT 


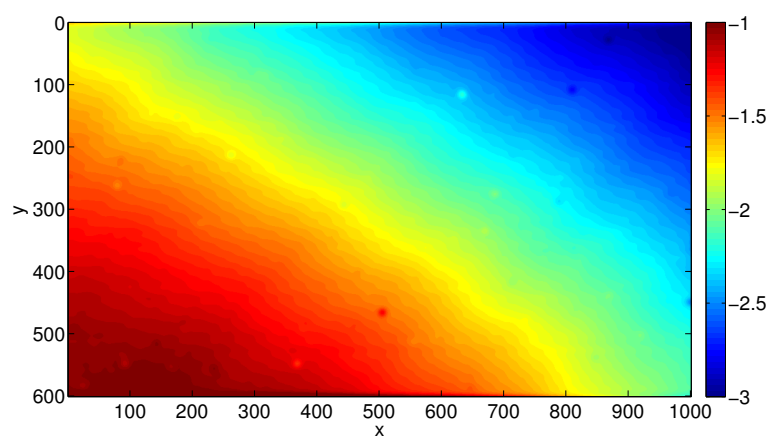

a- mean phase obtained with the raw grid images

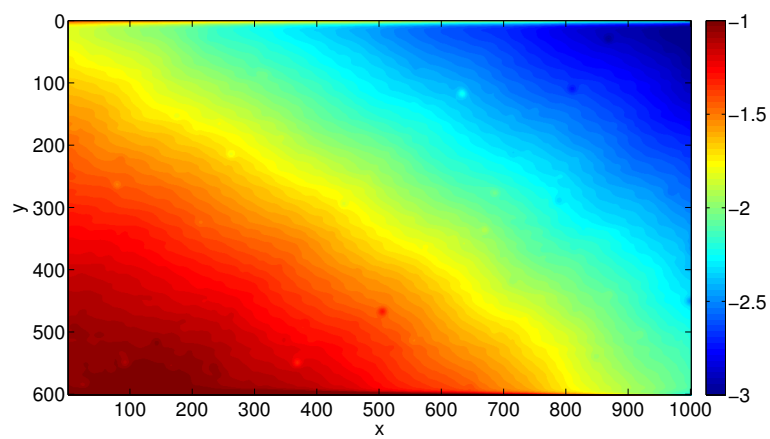

b- mean phase obtained with the normalised grid images

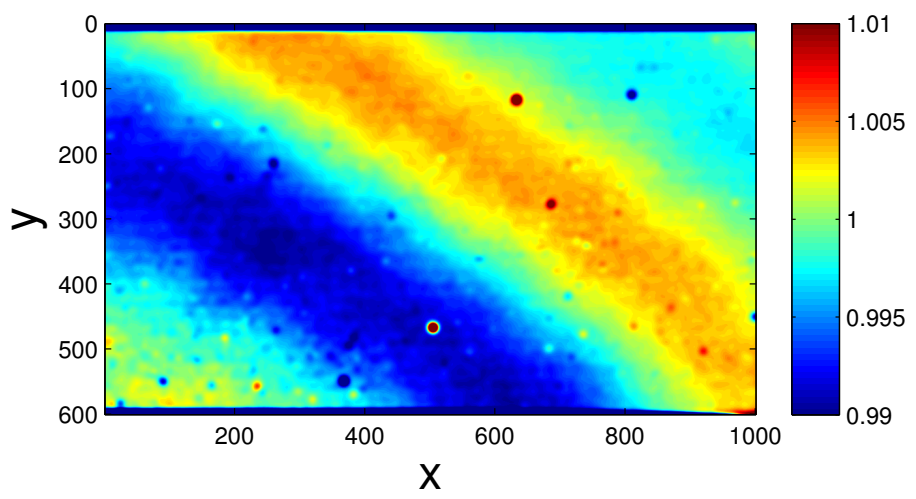

c- ratio between b- and a-

Figure 15: Comparison between the phase maps $\Phi_{y}$ obtained with the raw (a-) and normalised (b-) grid images 


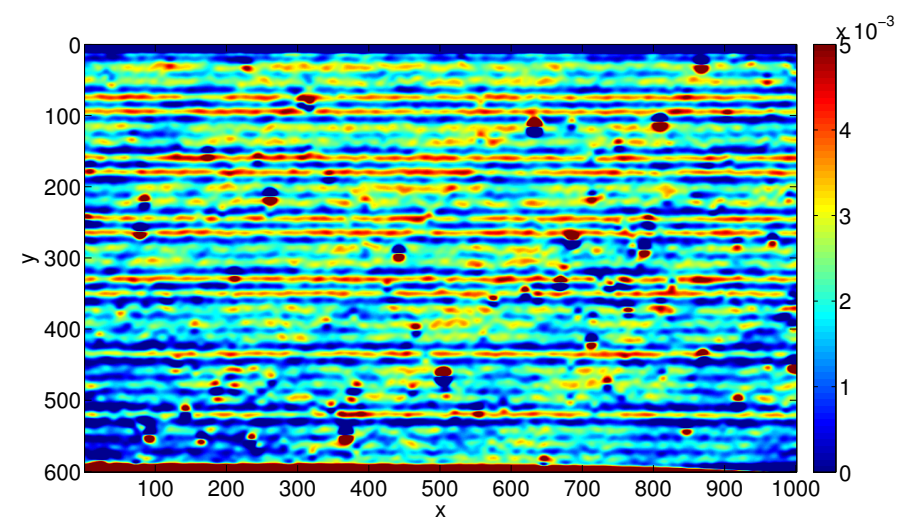

a- mean phase derivative obtained with the raw grid images

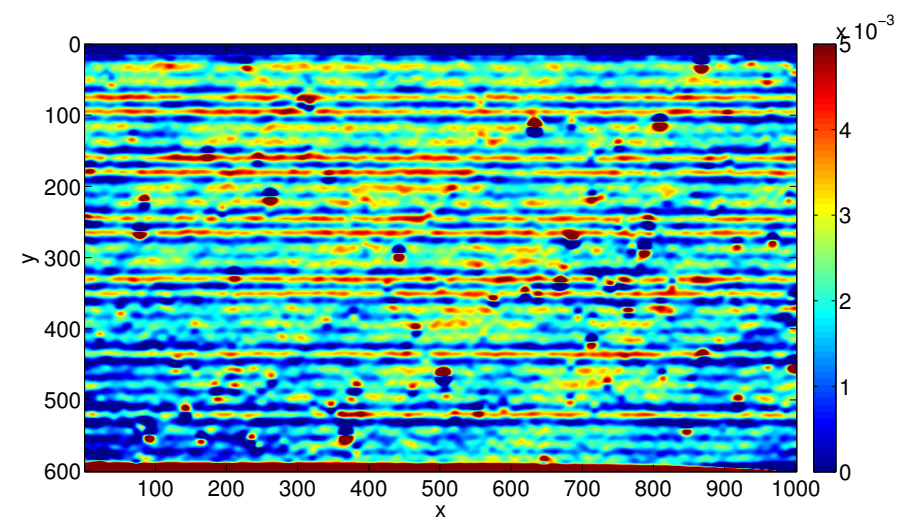

b- mean phase derivative obtained with the normalised grid images

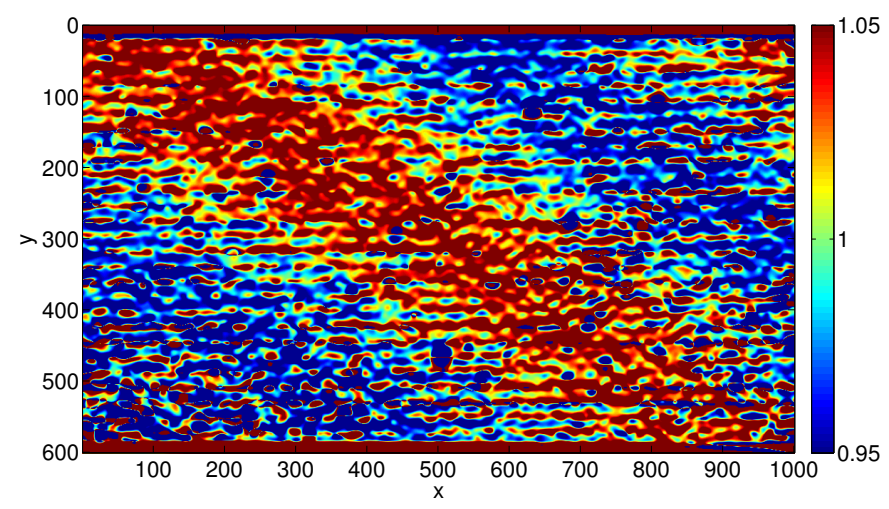

c- ratio between b- and a-

Figure 16: Comparison between the phase derivative maps $\frac{\partial \Phi_{y}}{\partial y}$ obtained with the raw (a-) and normalised (b-) grid images 


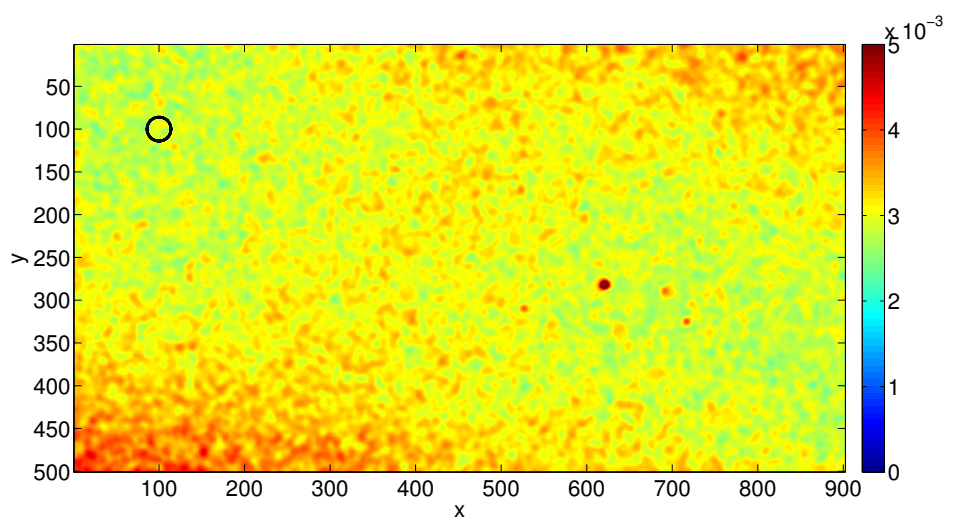

a- estimated with the grid images after NRSR and GAT

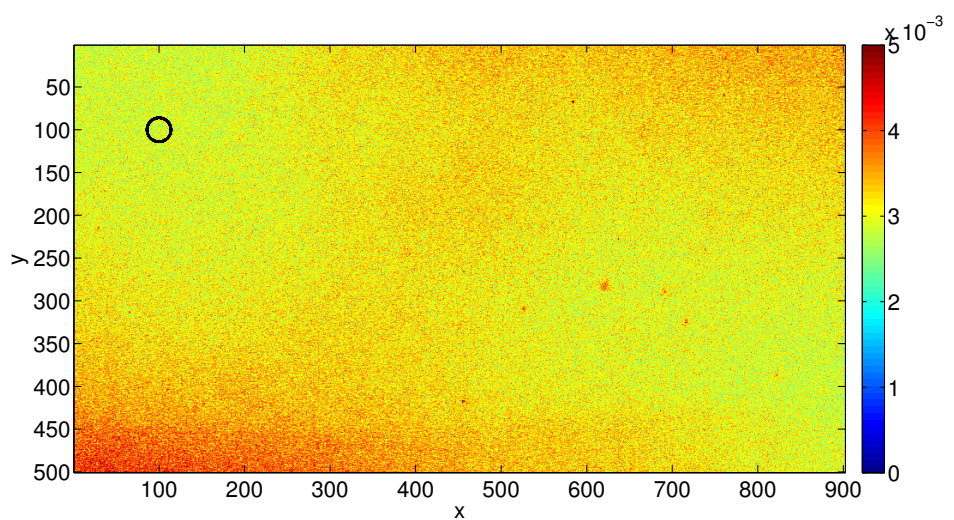

b- predicted with Equation 9-a

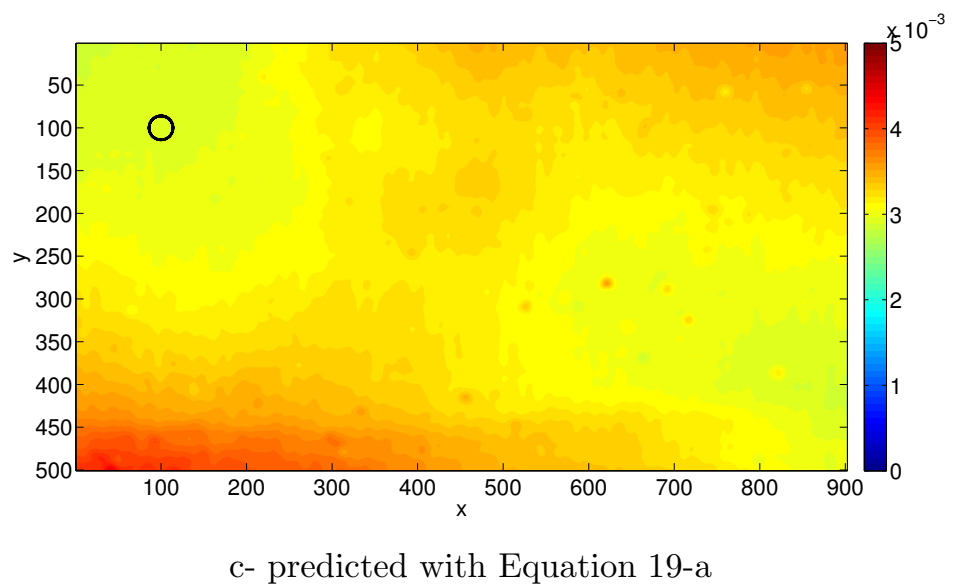

Figure 17: Phase resolution. The black circle at the top left has a diameter equal to the spatial resolution $d_{\phi}$ 


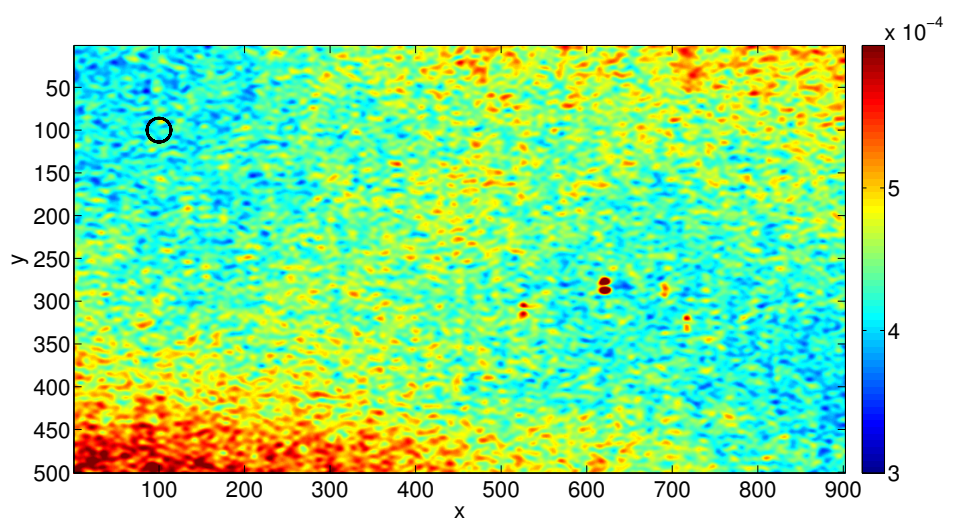

a- estimated with the grid images after NRSR and GAT

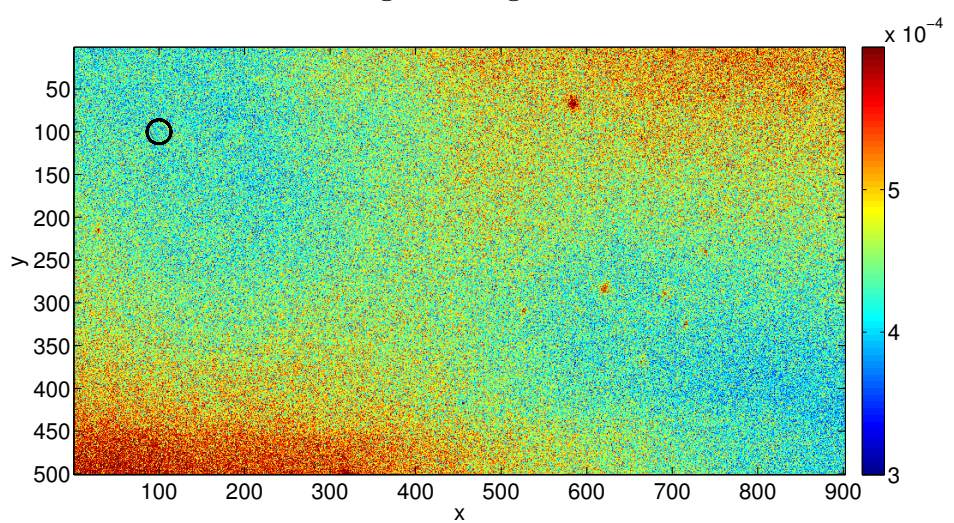

b- predicted with Equation 9-b

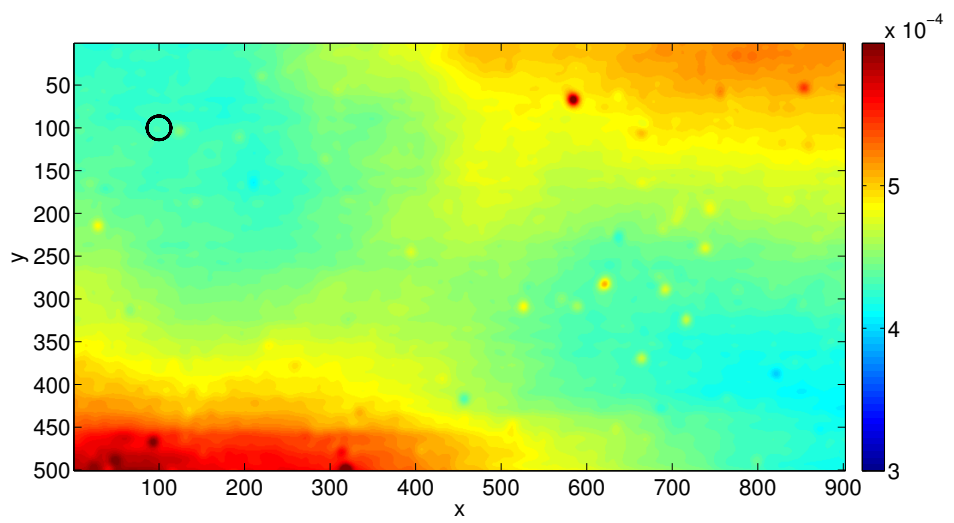

c- predicted with Equation 19-b

Figure 18: Phase derivative resolution. The black circle at the top left has a diameter equal to the spatial resolution $d_{\phi^{\prime}}$. 


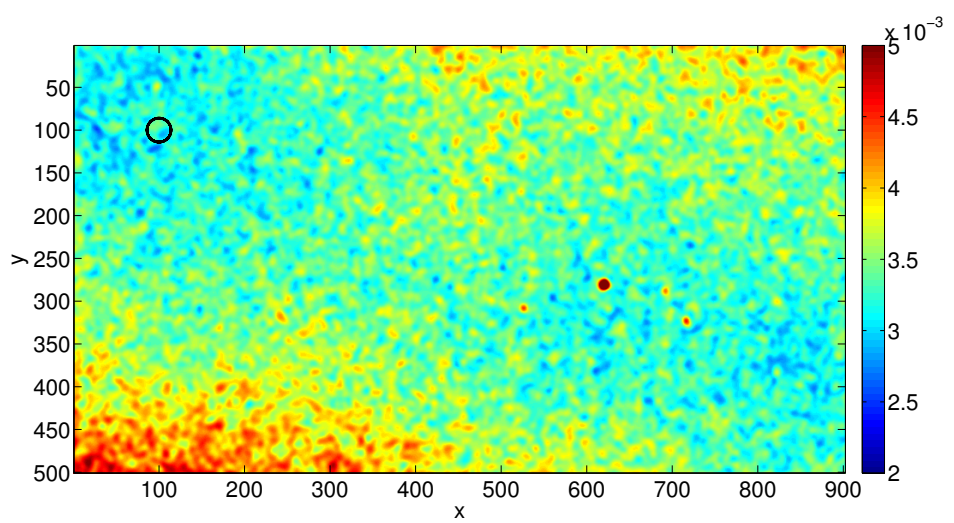

a- estimated with normalised grid images

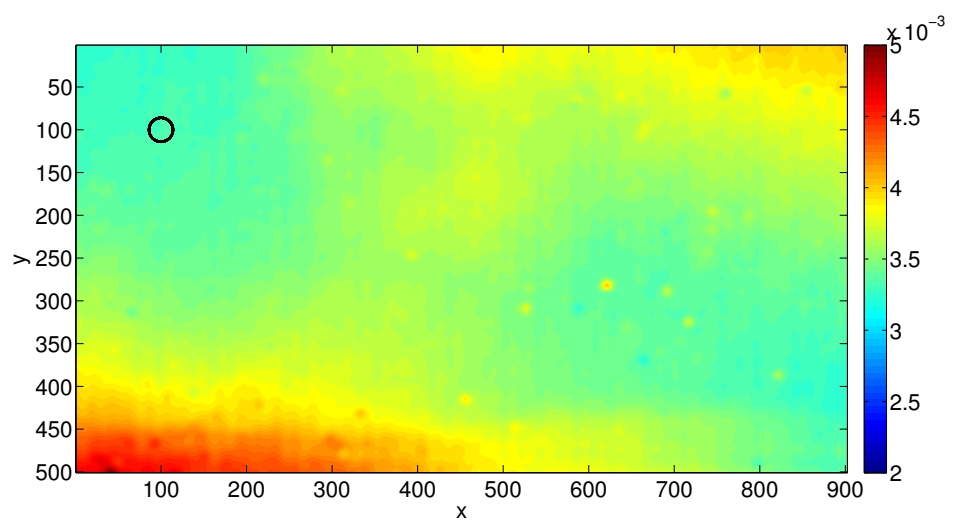

b- predicted with Equation 20-a

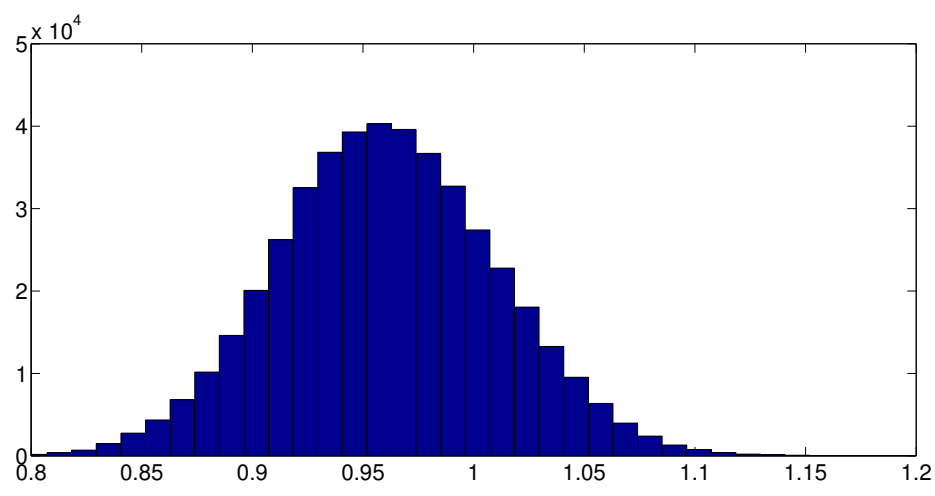

c- ratio $\alpha^{\prime}$ between displacement resolution identified with normalised grid images and predicted resolutions.

Mean value: 0.961

Figure 19: Displacement resolution in pixel (in micron by multiplying by 40). Imposed displacement $u=0.1 \mathrm{~mm}$. The black circle at the top left is the spatial resolution 


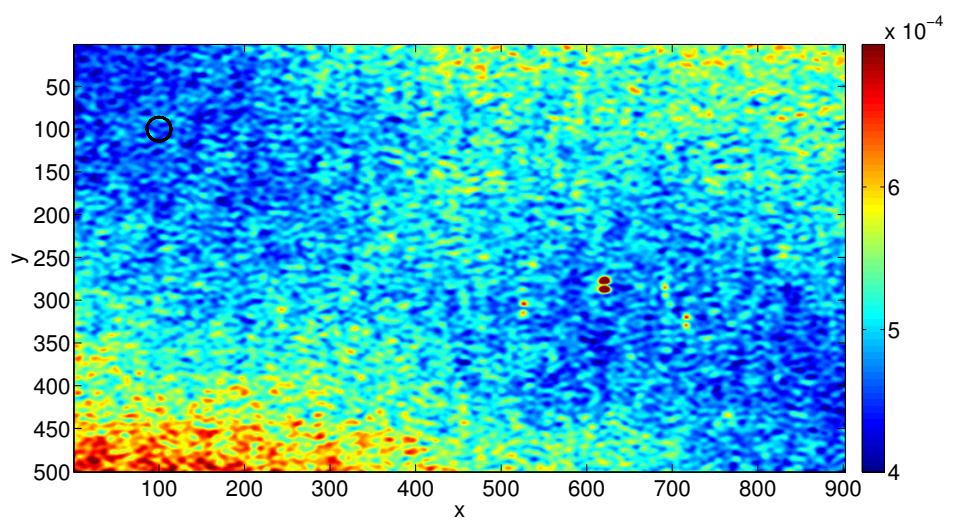

a- estimated with normalised grid images

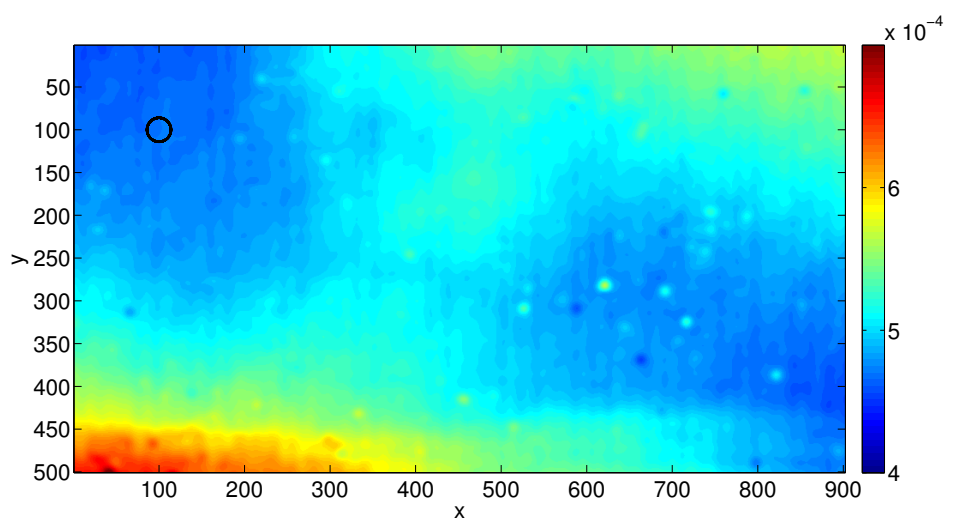

b- predicted with Equation 20-b

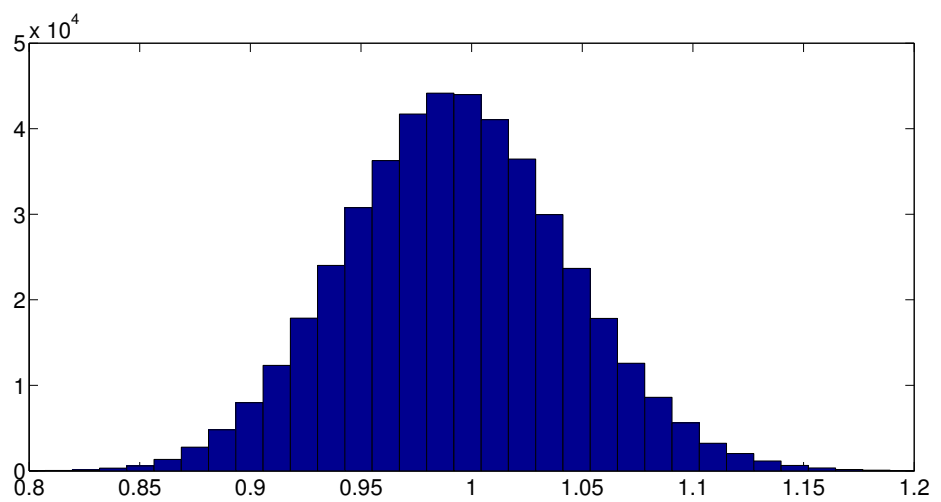

c- ratio $\beta^{\prime}$ between strain resolution identified

with normalised grid images and predicted resolutions.

Mean value: 0.993

Figure 20: Strain resolution. Imposed displacement $u=0.1 \mathrm{~mm}$. The black circle at the top left is the spatial resolution. 


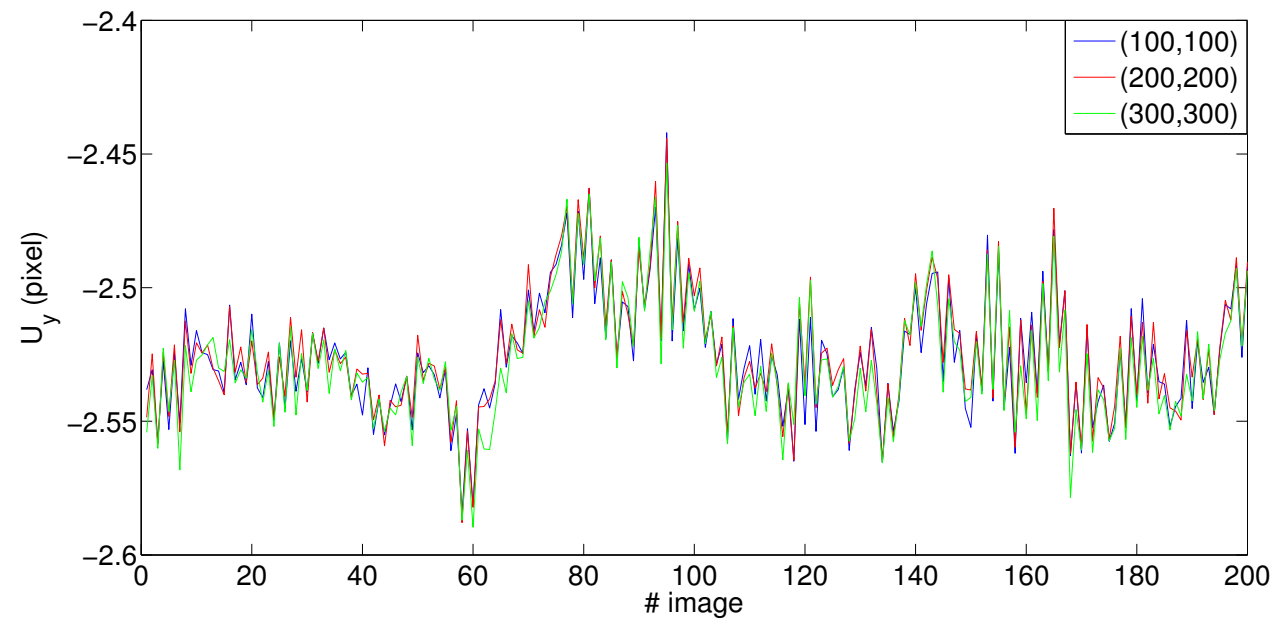

Figure 21: Vertical displacement (along y) at three different pixels: $(100,100),(200,200)$ and $(300,300)$. In pixel ( 1 pixel $=40$ micrometers) 


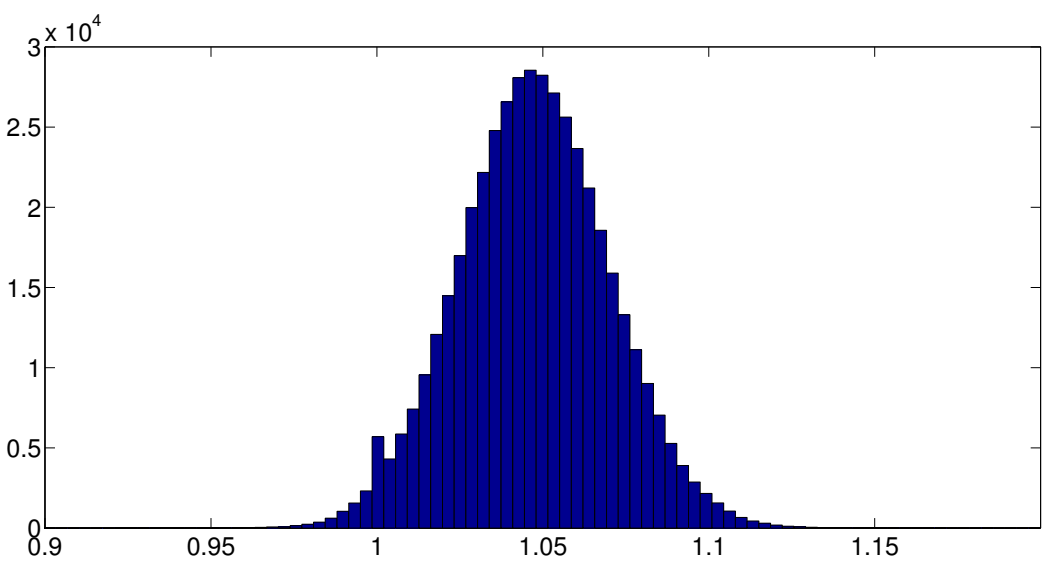

a- histogram of the ratio between strain resolution identified with normalised and raw grid images. Mean value: 1.048

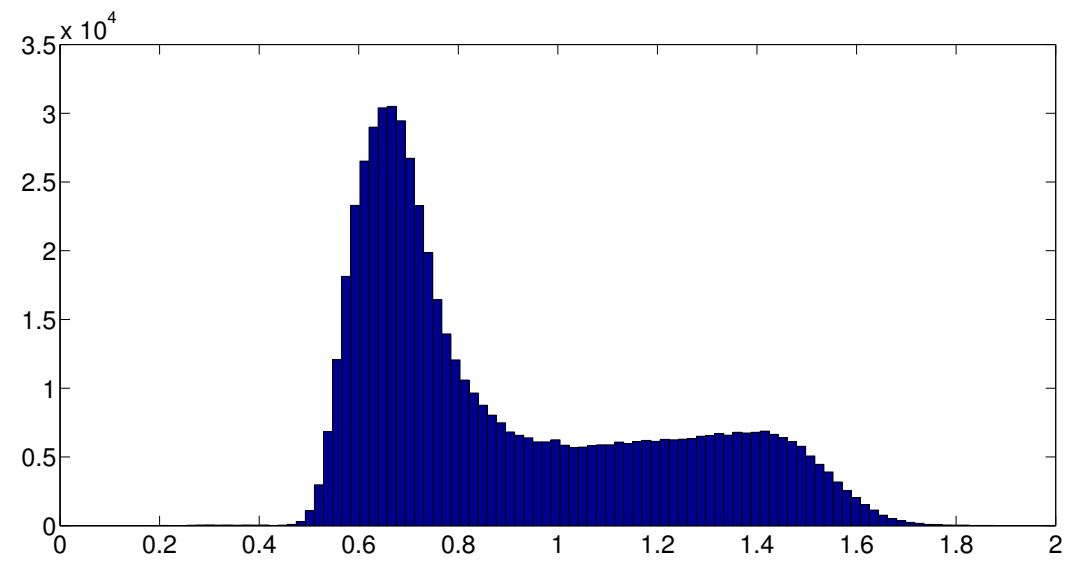

b- histogram of the ratio between strain resolution predicted with raw grid images and normalised grid images.

Mean value: 0.9017

Figure 22: Using raw grid images instead of normalised ones for the estimation of the strain resolution. Imposed displacement $u=0.1 \mathrm{~mm}$ 


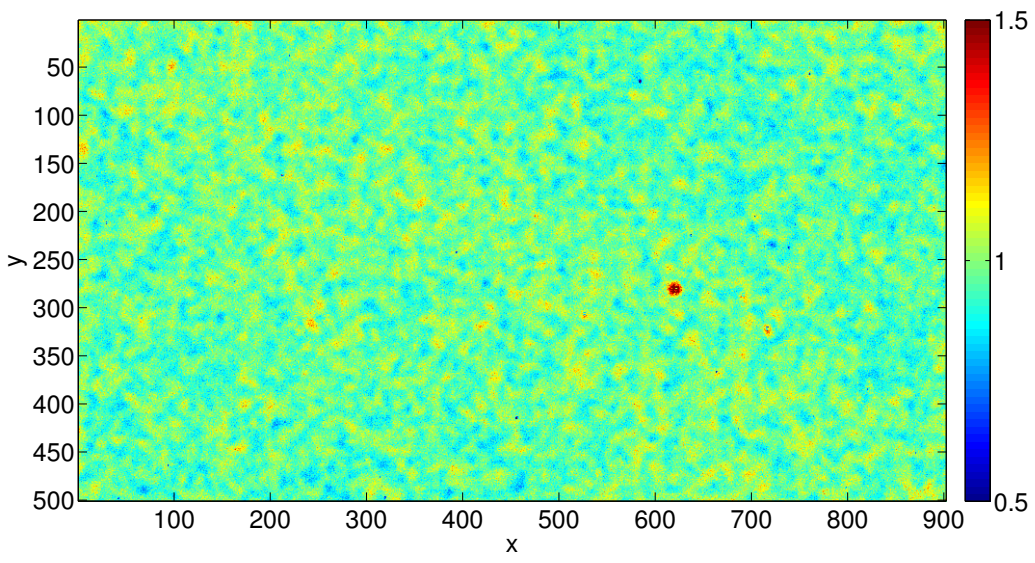

a- spatial distribution of the ratio $\alpha^{\prime}$ whose histogram is shown in Figure 19-c.

To be compared with the distribution of $\alpha$ in Figure 12-c which depends on brightness

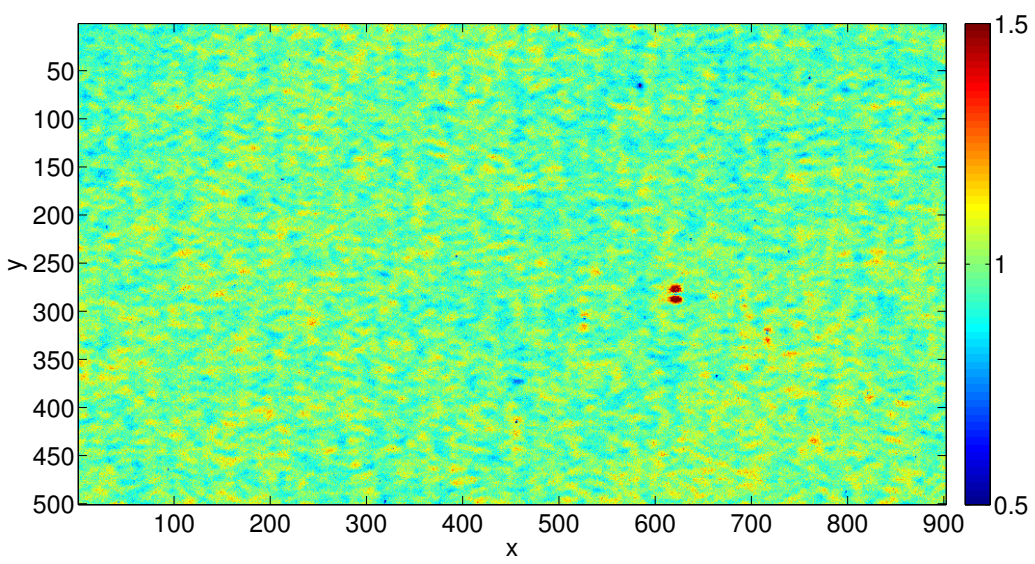

b- spatial distribution of the ratio $\beta^{\prime}$ whose histogram is shown in Figure 20-c.

To be compared with the distribution of $\beta$ in Figure 13-c which depends on brightness

Figure 23: Benefit of GAT in addition to NRSR: random spatial distribution around 1 of the ratio between identified and predicted resolutions 